\title{
LARGE DEVIATIONS, MODERATE DEVIATIONS, AND THE KLS CONJECTURE
}

\author{
DAVID ALONSO-GUTIÉRREZ, JOSCHA PROCHNO, AND CHRISTOPH THÄLE
}

\begin{abstract}
Having its origin in theoretical computer science, the Kannan-Lovász-Simonovits (KLS) conjecture is one of the major open problems in asymptotic convex geometry and high-dimensional probability theory today. In this work, we establish a connection between this conjecture and the study of large and moderate deviations for isotropic log-concave random vectors. We then study the moderate deviations for the Euclidean norm of random orthogonally projected random vectors in an $\ell_{p}^{n}$-ball. This leads to a number of interesting observations: (A) the $\ell_{1}^{n}$-ball is critical for the new approach; (B) for $p \geq 2$ the rate function in the moderate deviations principle undergoes a phase transition, depending on whether the scaling is below the square-root of the subspace dimensions or comparable; (C) for $1 \leq p<2$ and comparable subspace dimensions, the rate function again displays a phase transition depending on its growth relative to $n^{p / 2}$.
\end{abstract}

\section{Introduction ANd Results}

One of the major open problems in asymptotic convex geometry is the famous Kannan-Lovász-Simonovits (KLS) conjecture. This conjecture has its origin in theoretical computer science and arose in the study of sampling algorithms for high-dimensional convex bodies. One specific situation of interest in the theory is to design an algorithm that efficiently computes the volume of an $n$-dimensional convex body. Such an algorithm is fed with a convex body $K \subseteq \mathbb{R}^{n}$ and a quality parameter $\varepsilon \in(0, \infty)$, where the body $K$ is represented by a membership oracle, which, for each $x \in \mathbb{R}^{n}$ can decide whether or not $x \in K$. The algorithm returns a number $V=V(K, \varepsilon) \in \mathbb{R}$ such that

$$
(1-\varepsilon) \operatorname{vol}_{n}(K) \leq V \leq(1+\varepsilon) \operatorname{vol}_{n}(K)
$$

and the efficiency of the designed algorithm is measured in terms of the number of arithmetic operations and calls to the membership oracle. While deterministic algorithms are deemed to be inefficient (see the works of Bárány and Füredi [4] and of Elekes [14]), there are reasonable randomized algorithms available that can accurately compute the volume of convex bodies with high probability in polynomial time, see Dyer, Frieze, and Kannan [13]. At its core, the construction of this randomized algorithm is connected to an isoperimetric inequality for log-concave probability measures on $\mathbb{R}^{n}$ (see, e.g., [1] for an introduction to the KLS conjecture and [38] for a more detailed explanation on its connection with problems in theoretical computer science). The constant appearing in this isoperimetric inequality, known as Cheeger's constant, is of particular interest and directly linked to the KLS conjecture, which by the work of Rothaus, Cheeger, Maz'ya, and Ledoux (see, e.g., [7, Theorem 1.1]) can be stated as follows:

KLS Conjecture. There exists an absolute constant $C \in(0, \infty)$ such that for all $n \in \mathbb{N}$, every centered random vector $X$ with log-concave distribution and any locally Lipschitz function $f: \mathbb{R}^{n} \rightarrow \mathbb{R}$ such that $f(X)$ is of finite variance,

where $\lambda_{X}^{2}:=\sup _{\theta \in \mathbb{S}^{n-1}} \mathbb{E}\langle X, \theta\rangle^{2}$.

$$
\operatorname{Var}[f(X)] \leq C \lambda_{X}^{2} \mathbb{E}\left[\|\nabla f(X)\|_{2}^{2}\right]
$$

Using what is known as the localization lemma, Kannan, Lovász, and Simonovits proved the conjecture with a factor $\left(\mathbb{E}\|X\|_{2}\right)^{2}$ instead of $\lambda_{X}^{2}$ (see [26]), and later Bobkov [9] improved this estimate. But besides the fact that the KLS conjecture holds for exponential and Gaussian random vectors and has attracted considerable attention in the past 25 years, there has been very little progress to the present day (see [8] and [19] for a proof of the conjecture for the class of revolution bodies, [6] for the case of the simplex, [30] and [36] for the

2010 Mathematics Subject Classification. Primary: 60F10, 52A23 Secondary: 60D05, $46 B 09$.

Key words and phrases. Asymptotic geometric analysis, convex bodies, $\ell_{p}^{n}$-balls, KLS conjecture, large deviations principle, moderate deviations principle, random projections, stochastic geometry. 
case of the $\ell_{p}^{n}$-balls, [29] for the case of 1-unconditional bodies with an extra $\log n$ factor, and [32] for the best general estimate of the constant of the order $\sqrt{n}$ in the KLS conjecture). One of the reasons for the interest in the KLS conjecture is that, if correct, it would imply several other well-known conjectures in asymptotic convex geometry, including the variance conjecture (which is the special case $f(\cdot)=\|\cdot\|_{2}$ ) and the hyperplane conjecture. This brings us to the first purpose of this paper, which is to establish a connection between the KLS conjecture and the study of so-called large and moderate deviations principles for isotropic log-concave random vectors.

While the study of central limit theorems for random geometric quantities in asymptotic convex geometry is by now a classical and still flourishing part of the theory, as can be seen in [3, 18, 20,21,22, 23, 28, 34, 35], fluctuations beyond the Gaussian scale have only recently attracted attention in this context. For one, there are the large deviations, or more precisely large deviations principles, which determine the asymptotic likelihood of rare events on a scale of a law of large numbers, the most classical result in this direction being Cramér's theorem [10] (see also [12]). Contrary to the universality shown by a central limit theorem, the large deviation behavior is typically sensitive to the distribution of the underlying random objects. Being a classical topic in probability theory, large deviations have only recently been introduced in asymptotic convex geometry and high-dimensional probability by Gantert, Kim, and Ramanan [15]. They obtained a large deviations principle for 1-dimensional projections of $\ell_{p}^{n}$-balls in $\mathbb{R}^{n}$ as the space dimension $n$ tends to infinity, showing how the speed and rate depend on the parameter $p$ and thus in a subtle way on the geometry of the body. Their work inspired a number of investigations regarding the large deviations behavior of quantities that naturally appear in asymptotic convex geometry, such as Sanov-type large deviations for the random spectral measure of a properly normalized matrix chosen uniformly at random in the unit ball of a Schatten $p$-class [24] (a non-commutative analogue of a result previously obtained by Kim and Ramanan for random vectors in high-dimensional $\ell_{p}^{n}$-spheres [27]), large deviations principles for $\ell_{q}$-norms of random vectors in $\ell_{p}^{n}$ balls $[22,23]$, a description of the large deviations behavior for the Euclidean norm of orthogonal projections of $\ell_{p}^{n}$-balls to high-dimensional random subspaces [2], and, very recently, large deviations under an asymptotic thin-shell condition [37] as well as geometric sharp large deviations for random projections of $\ell_{p}^{n}$-spheres [33]. The question now arises of what can be said about the asymptotic likelihood of events on a scaling between Gaussian fluctuations covered by the central limit theorem and large deviations? This scaling is exactly the one covered by the so-called moderate deviations principles, which are nothing but large deviations principles of differently normalized random variables. However, in contrast to large deviations, the so-called moderate deviations principles are typically non-parametric in rate. In fact, usually moderate deviations principles inherit properties from both the central limit theorem and the large deviations principle in that the central limit theorem is still visible in form of a Gaussian (quadratic) rate function, while probabilities decay on an exponential scale as in the large deviations principle. In the framework of random geometric quantities in asymptotic convex geometry those have first been considered by Kabluchko, Prochno, and Thäle in [23] for $\ell_{q}$-norms of random vectors in $\ell_{p}^{n}$-balls.

Now, let us be more precise and introduce what a large or moderate deviations principle formally is and then describe its relation to the KLS conjecture. We recall that a sequence $\left(X_{n}\right)_{n \in \mathbb{N}}$ of random vectors in $d$-dimensional Euclidean space $\mathbb{R}^{d}$ satisfies a large deviations principle (LDP) with speed $s_{n}$ and rate function $\mathbb{I}: \mathbb{R}^{d} \rightarrow[0, \infty]$ if

$$
-\inf _{x \in A^{\circ}} \mathbb{I}(x) \leq \liminf _{n \rightarrow \infty} s_{n}^{-1} \log \mathbb{P}\left[X_{n} \in A\right] \leq \limsup _{n \rightarrow \infty} s_{n}^{-1} \log \mathbb{P}\left[X_{n} \in A\right] \leq-\inf _{x \in \bar{A}} \mathbb{I}(x)
$$

for all Borel measurable $A \subseteq \mathbb{R}^{d}$, where $A^{\circ}$ denotes the interior and $\bar{A}$ the closure of $A$, and where $\mathbb{I}$ is lower semi-continuous and has compact level sets $\left\{x \in \mathbb{R}^{d}: \mathbb{I}(x) \leq \alpha\right\}, \alpha \in \mathbb{R}$. So loosely speaking, an LDP says that if $n \in \mathbb{N}$ is sufficiently large and $A \subseteq \mathbb{R}^{d}$ sufficiently regular, then

$$
\mathbb{P}\left[X_{n} \in A\right] \approx e^{-s_{n}} \inf _{x \in A} \mathbb{I}(x) .
$$

As mentioned before, a moderate deviations principle (MDP) is nothing else than an LDP for a differently normalized sequence of random variable. Namely, if a sequence $\left(t_{n}^{-1} X_{n}\right)_{n \in \mathbb{N}}$ of random variables satisfies an LDP with some speed and some rate function, an LDP for the sequence random variables $\tilde{t}_{n}^{-1} X_{n}$ with $\tilde{t}_{n} \rightarrow \infty$ and $\tilde{t}_{n}=o\left(t_{n}\right)$ is referred to as a moderate deviations principle, see [11, Chapter 3.7] and especially part (c) of the remark after Theorem 3.7.1 for further details on terminology. Typically, the reference to an LDP corresponds to the scaling that provides deviations at the scale of a law of large numbers, as Cramér's theorem does for sums of independent random variables, and LDPs at the scales providing deviations between 
that of a weak limit theorem (like a central limit theorem) and that of a law of large numbers are referred to as MDPs. In this paper, for two sequences $\left(x_{n}\right)_{n \in \mathbb{N}}$ and $\left(y_{n}\right)_{n \in \mathbb{N}}$ we use the Landau notation $x_{n}=o\left(y_{n}\right)$ if $\lim _{n \rightarrow \infty} \frac{x_{n}}{y_{n}}=0$ and $x_{n}=\omega\left(y_{n}\right)$ if $\lim _{n \rightarrow \infty}\left|\frac{x_{n}}{y_{n}}\right|=+\infty$. In addition, we write $x_{n} \approx y_{n}$ if there exist two absolute constants $c_{1}, c_{2}$ such that $c_{1} x_{n} \leq y_{n} \leq c_{2} x_{n}$. The following result establishes a connection between the KLS conjecture and moderate/large deviations principles. Essentially, it is a consequence of the fact, known from $[1,17,31]$, that the KLS conjecture would imply a sharp concentration bound for the Euclidean norm of isotropic log-concave random vectors.

Theorem A (LDPs/MDPs and the KLS conjecture). Let $1 \leq k_{n} \leq n$ be a sequence of integers such that $k_{n}=\omega(1)$ and let $\left(\xi_{n}\right)_{n=1}^{\infty}$ be a sequence of isotropic log-concave random vectors in $\mathbb{R}^{k_{n}}$. Consider a sequence of random variables $X_{n}=\frac{\left\|\xi_{n}\right\|_{2}}{\sqrt{k_{n}}}, n \in \mathbb{N}$, which satisfies (1) with speed $s_{n}$ and rate function $\mathbb{I}$. Assume that one of the following two conditions is satisfied:

(a) $s_{n}=o\left(\sqrt{k_{n}}\right)$ and $\mathbb{I}$ is non-singular, i.e., $\mathbb{I}(x) \neq \mathbb{I}_{0}(x)= \begin{cases}0 & : x=1 \\ +\infty & : \text { otherwise. }\end{cases}$

(b) $s_{n} \approx \sqrt{k_{n}}$ and $\inf _{t>t_{0}} \frac{\inf _{x \in(t, \infty)} \mathbb{I}(x)}{t}=0$ for some absolute constant $t_{0} \in(1, \infty)$.

Then the KLS conjecture is false.

For a sequence of random vectors $\xi_{n} \in \mathbb{R}^{k_{n}}, n \in \mathbb{N}$, as given in Theorem $\mathrm{A}$ it is well known that $\mathbb{E}\left\|\xi_{n}\right\|_{2} / \sqrt{k_{n}} \rightarrow$ 1 , as $n \rightarrow \infty$. Against this light, the natural scale for a law of large numbers and hence an LDP is $\sqrt{k_{n}}$, while scales with $t_{n}=o\left(\sqrt{k_{n}}\right)$ and $t_{n}=\omega(1)$ correspond to an MDP. Thus, Theorem A establishes a connection between LDPs or MDPs for isotropic log-concave random vectors and the KLS conjecture, showing that by constructing a sequence of random vectors $\xi_{n}, n \in \mathbb{N}$, such that $X_{n}, n \in \mathbb{N}$, follows an LDP or MDP with an appropriate speed and/or rate function would disprove the variance and hence the KLS conjecture.

In this context it is instructive to recall that the large deviations principle [2, Theorem 1.2] or [22, Theorem D] for the Euclidean norm of uniform random points in the rescaled $n$-dimensional crosspolytope $\mathbb{D}_{1}^{n}:=$ $\sqrt{\frac{(n+1)(n+2)}{2}} \mathbb{B}_{1}^{n}$ (the normalization is designed in such a way that a uniform random vector in $\mathbb{D}_{1}^{n}$ is isotropic) holds with $s_{n}=\sqrt{n}$ and rate function

$$
\mathbb{I}(x)= \begin{cases}\sqrt{x^{2}-1} & : x>1 \\ +\infty & : \text { otherwise }\end{cases}
$$

In fact, the result from [2] can be transferred to our rescaled situation by a contraction with the function $x \mapsto x / \sqrt{2}$. This implies that, for any $t_{0}>1$, the function

$$
\frac{\sqrt{t^{2}-1}}{t}=\sqrt{1-\frac{1}{t^{2}}}
$$

is equivalent to a constant in the interval $\left[t_{0}, \infty\right)$, since

$$
\inf _{t>t_{0}} \sqrt{1-\frac{1}{t^{2}}}=\sqrt{1-\frac{1}{t_{0}^{2}}} \text { and } \sup _{t>t_{0}} \sqrt{1-\frac{1}{t^{2}}}=1 .
$$

Thus, we cannot find $t_{0}>1$ such that the condition in Theorem $\mathrm{A}(\mathrm{b})$ is satisfied while at the same time there exists no $t_{0}>1$ and an exponent $\alpha>1$ such that $\inf _{t>t_{0}} \frac{\inf _{x \in(t, \infty)} \mathbb{I}(x)}{t^{\alpha}}>C$ for some absolute constant $C \in(0, \infty)$. This shows that (rescaled) crosspolytopes constitute a critical case in Theorem A as they just fail to satisfy condition (b) there. Notice also that the case of the crosspolytope shows that the condition $t_{0} \in(1, \infty)$ in (b) cannot be relaxed to $t_{0} \in[1, \infty)$ as this would give $\inf _{t>1} \frac{\inf _{x \in(t, \infty)} \mathbb{I}(x)}{t}=0$ while we know that they do satisfy the KLS conjecture (see [1, Theorem 2.3]).

The crosspolytopes just discussed are members of the important class of $\ell_{p}^{n}$-balls, whose probabilistic aspects have been under intensive investigation during the last years $[2,3,15,16,20,22,23,27,33,37$ ] (see also the comments in the introduction). As usual, for $1 \leq p<\infty$ we let $\mathbb{B}_{p}^{n}:=\left\{x \in \mathbb{R}^{n}:\|x\|_{p} \leq 1\right\}$ denote the unit ball in $\mathbb{R}^{n}$ with respect to the $\ell_{p}^{n}$-norm $\|x\|_{p}:=\left(\left|x_{1}\right|^{p}+\ldots+\left|x_{n}\right|^{p}\right)^{1 / p}, x=\left(x_{1}, \ldots, x_{n}\right) \in \mathbb{R}^{n}$. In the remaining parts of this paper we are studying random vectors that arise as orthogonal projections of uniformly distributed random points in $\mathbb{B}_{p}^{n}$ onto random $k_{n}$-dimensional subspaces. To formally introduce 
the framework and the necessary notation, let $\left(k_{n}\right)_{n \in \mathbb{N}}$ be a sequence of integers with $1 \leq k_{n} \leq n$. Further, for each $n \in \mathbb{N}$ let $X_{n}$ be a uniformly distributed random vector in $\mathbb{B}_{p}^{n}$. Consider a random $k_{n}$-dimensional subspace $E_{n}$ of $\mathbb{R}^{n}$, which is distributed according to the Haar probability measure on the Grassmannian manifold of all $k_{n}$-dimensional subspaces of $\mathbb{R}^{n}$ and assumed to be independent of $X_{n}$. By $P_{E_{n}}$ we denote the orthogonal projection onto $E_{n}$ and introduce the random variables

$$
\mathscr{Z}_{n, p}:=n^{1 / p}\left\|P_{E_{n}} X_{n}\right\|_{2}, \quad n \in \mathbb{N},
$$

where the factor $n^{1 / p}$ is motivated by the asymptotic order of the factor $\operatorname{vol}\left(\mathbb{B}_{p}^{n}\right)^{1 / n}$ by which one has to divide $X_{n}$ in order to make it, and consequently $P_{E_{n}} X_{n}$, isotropic. In [15] and [2], large deviations principles were proved (in some cases) for the sequence of random variables $n^{-1 / 2} \mathscr{Z}_{n, p}$. While these result provide large deviations principles at the scale $\sqrt{k_{n}}$ whenever $k_{n}$ is proportional to $n$, that is not the case if $k_{n}=o(n)$. For that matter, we will use the terminology MDP instead of LDP whenever we look at scales below $\sqrt{n}$ and call $\sqrt{k_{n}}$ the critical scale. Our next result is an MDP for $\mathscr{Z}_{n, p}$ on the critical scale $\sqrt{k_{n}}$, i.e., for $k_{n}^{-1 / 2} \mathscr{Z}_{n, p}$, whose study is motivated by Theorem A. We will see that in this particular situation the rate function is neither universal nor given by the quadratic Gaussian rate function. Instead, the rate function for $1 \leq p<2$ displays an interesting and non-expected phase transition, which is controlled by the relative growth of the subspace dimensions $k_{n}$ to $n^{p / 2}$.

Theorem B (MDP on the critical scale). Let $1 \leq p<\infty$ and $\left(k_{n}\right)_{n \in \mathbb{N}}$ be a sequence of positive integers $1 \leq k_{n} \leq n$ such that $k_{n}=\omega(1)$ and $k_{n}=o(n)$. Let $M_{p}(2)=\frac{p^{2 / p}}{3} \frac{\Gamma\left(1+\frac{3}{p}\right)}{\Gamma\left(1+\frac{1}{p}\right)}$. Then the following hold:

(a) If $p \geq 2$, then $k_{n}^{-1 / 2} \mathscr{Z}_{n, p}$ satisfies an MDP on $\mathbb{R}$ with speed $k_{n}$ and rate function

$$
\mathbb{I}(x)= \begin{cases}\frac{x^{2}-M_{p}(2)}{2 M_{p}(2)}-\log \frac{x}{\sqrt{M_{p}(2)}} & : x>0 \\ +\infty & : \text { otherwise. }\end{cases}
$$

(b1) If $1 \leq p<2$ and $k_{n}=o\left(n^{p / 2}\right)$, then $k_{n}^{-1 / 2} \mathscr{Z}_{n, p}$ satisfies an MDP on $\mathbb{R}$ with speed $k_{n}$ and rate function

$$
\mathbb{I}(x)= \begin{cases}\frac{x^{2}-M_{p}(2)}{2}-\log \frac{x}{\sqrt{M_{p}(2)}} & : x>0 \\ +\infty & : \text { otherwise. }\end{cases}
$$

(b2) If $1 \leq p<2$ and $k_{n}=n^{p / 2}$, then $k_{n}^{-1 / 2} \mathscr{Z}_{n, p}$ satisfies an $M D P$ on $\mathbb{R}$ with speed $n^{p / 2}$ and rate function

$$
\mathbb{I}(x)= \begin{cases}\inf _{y \geq m_{p}} \frac{\left(\frac{x}{y}\right)^{2}-1}{2}-\log \left(\frac{x}{y}\right)+\frac{1}{p}\left(y^{2}-M_{p}(2)\right)^{\frac{p}{2}} & : x>0 \\ +\infty & : \text { otherwise. }\end{cases}
$$

(b3) If $1 \leq p<2$ and $k_{n}=\omega\left(n^{p / 2}\right)$, then $k_{n}^{-1 / 2} \mathscr{Z}_{n, p}$ satisfies an MDP on $\mathbb{R}$ with speed $n^{p / 2}$ and rate function

$$
\mathbb{I}(x)= \begin{cases}\frac{1}{p}\left(x^{2}-M_{p}(2)\right)^{\frac{p}{2}} & : x>\sqrt{m_{p}} \\ +\infty & : \text { otherwise }\end{cases}
$$

After having discussed the moderate deviations at the critical scale $\sqrt{k_{n}}$, we finally turn to a description of the moderate deviations at subcritical scales $o\left(\sqrt{k_{n}}\right)$, which complement the central limit theorem and the large deviations principle obtained in [3] and [2], respectively. In this case, we will see that the rate function is indeed the universal quadratic Gaussian rate function also visible in the classical central limit theorem, but with a typical prefactor encoding the variance of the Gaussian random variable appearing on the scale of the central limit theorem as the limit of the sequence of random variables $\mathscr{X}_{n, p}$, defined for every $n \in \mathbb{N}$ as

$$
\mathscr{X}_{n, p}:=n^{1 / p} \sqrt{\frac{\Gamma\left(\frac{1}{p}\right)}{p^{2 / p} \Gamma\left(\frac{3}{p}\right)}}\left\|P_{E_{n}} X_{n}\right\|_{2}-\sqrt{k_{n}},
$$

which is normalized in such a way that $\mathbb{E}\left[\mathscr{X}_{n, p}\right] \rightarrow 0$, as $n \rightarrow \infty$. For technical reasons, we also restrict the following result to the case that $p \geq 2$. 
Theorem C (MDP on subcritical scales). Let $2 \leq p<\infty$ and $\left(k_{n}\right)_{n \in \mathbb{N}}$ be a sequence of integers such that $1 \leq k_{n} \leq n$ and $\lim _{n \rightarrow \infty} \frac{k_{n}}{n}=\lambda \in[0,1]$. Assume that $k_{n}=\omega(1)$ and that the sequence $\left(t_{n}\right)_{n \in \mathbb{N}}$ of positive real numbers satisfies $t_{n}=\omega(1), t_{n}=o\left(\sqrt{k_{n}}\right)$ and either $t_{n}=o\left(\sqrt{n-k_{n}}\right)$ or $\left(n-k_{n}\right)=o\left(\frac{\sqrt{k_{n}}}{t_{n}}\right)$. Then the sequence of random variables $t_{n}^{-1} \mathscr{X}_{n, p}$ satisfies an $M D P$ on $\mathbb{R}$ with speed $t_{n}^{2}$ and rate function $\mathbb{I}(x)=\alpha_{p, \lambda} x^{2}$ with constant $\alpha_{p, \lambda}$ given by

$$
\alpha_{p, \lambda}=\frac{2 \Gamma\left(\frac{1}{p}\right) \Gamma\left(\frac{3}{p}\right)^{2}}{(2 p-\lambda(4+3 p)) \Gamma\left(1+\frac{1}{p}\right) \Gamma\left(\frac{3}{p}\right)^{2}+\lambda \Gamma\left(\frac{1}{p}\right)^{2} \Gamma\left(\frac{5}{p}\right)} .
$$

Remark 1.1. We end this section with a number of comments.

(a) Although the results we presented so far were formulated for uniform random vectors $X_{n}$ in $\mathbb{B}_{p}^{n}$, we will prove more general versions for random vectors that are distributed in $\mathbb{B}_{p}^{n}$ according to a distribution from the class introduced in [5]. As special cases this includes the uniform probability measure or the cone probability measure on $\mathbb{B}_{p}^{n}$, but also image measures of these measures on higher-dimensional $\ell_{p}^{n}$-balls under coordinate projections. We refer to the discussion in the next section.

(b) We would like to point out that the constant $\alpha_{p, \lambda}$ in (2) coincides with $\frac{1}{2 \sigma^{2}(p, \lambda)}$, where $\sigma^{2}(p, \lambda)$ is the variance of the centered Gaussian random variable appearing as the limit in the central limit theorem for $\mathscr{X}_{n, p}$ from [3]. In other words, this means that the rate function $\mathbb{I}(y)$ in Theorem $\mathrm{C}$ can be rewritten as $\mathbb{I}(y)=\frac{y^{2}}{2 \sigma^{2}(p, \lambda)}$, as expected.

(c) We leave it as an open problem for future research to study moderate deviations at the supercritical scales $t_{n}=\omega\left(\sqrt{k_{n}}\right)$ (and $t_{n}=o(\sqrt{n})$ ). In addition, it would be interesting to investigate the situation where the involved parameters neither satisfy $t_{n}=o\left(\sqrt{n-k_{n}}\right)$ nor $\left(n-k_{n}\right)=o\left(\frac{\sqrt{k_{n}}}{t_{n}}\right)$. Another open problem is to decide whether or not the rate function in the MDP in Theorem $\mathrm{C}$ is the same also for $1 \leq p<2$.

\section{Preliminaries}

2.1. Notation. We supply the $n$-dimensional Euclidean space $\mathbb{R}^{n}$, where $n \in \mathbb{N}$, with the Euclidean norm $\|\cdot\|_{2}$ and the standard scalar product $\langle\cdot, \cdot\rangle$. The $\ell_{p}^{n}$-norm will be denoted by $\|\cdot\|_{p}$ and $\mathbb{B}_{p}^{n}:=\left\{x \in \mathbb{R}^{n}\right.$ : $\left.\|x\|_{p} \leq 1\right\}$ will denote the $n$-dimensional unit ball with respect to the $\ell_{p}^{n}$-norm. For a set $A \subset \mathbb{R}^{d}$ we shall write $A^{\circ}$ for the interior and $\bar{A}$ for the closure of $A$.

2.2. Distributions on $\ell_{p}^{n}$-balls. As already mentioned in Remark 1.1, we consider a much more general class of distributions compared to [2], [15], [22] and [27]. Those have been intensively studied by Barthe, Guédon, Mendelson, and Naor [5], and are closely related to the geometry of $\ell_{p}^{n}$-balls. This class contains the uniform distribution as well as the cone probability measure on the $\ell_{p}^{n}$-unit ball $\mathbb{B}_{p}^{n}:=\left\{x \in \mathbb{R}^{n}:\|x\|_{p} \leq 1\right\}$ considered in $[3,15,22]$ as special cases, and many more (see below). As usual, $\|x\|_{p}:=\left(\left|x_{1}\right|^{p}+\ldots+\left|x_{n}\right|^{p}\right)^{1 / p}$ denotes the $\ell_{p}^{n}$-norm of the vector $x=\left(x_{1}, \ldots, x_{n}\right) \in \mathbb{R}^{n}$, and the parameter $p$ satisfies $1 \leq p<\infty$. For fixed space dimension $n \in \mathbb{N}$, we let $\mathbf{W}$ be any Borel probability measure on $[0, \infty), \mathbf{U}_{n, p}$ be the uniform distribution on $\mathbb{B}_{p}^{n}$, and $\mathbf{C}_{n, p}$ be the cone probability measure on the boundary of $\mathbb{B}_{p}^{n}$, where we recall that $\mathbf{C}_{n, p}(A):=\mathbf{U}_{n, p}(\{r x: x \in A, 0 \leq r \leq 1\})$ for a measurable subset $A$ on the boundary $\mathbb{S}_{p}^{n-1}=\left\{x \in \mathbb{R}^{n}\right.$ : $\left.\|x\|_{p}=1\right\}$ of $\mathbb{B}_{p}^{n}$. The distributions we consider are of the form

$$
\mathbf{P}_{\mathbf{W}, n, p}:=\mathbf{W}(\{0\}) \mathbf{C}_{n, p}+H \mathbf{U}_{n, p},
$$

where the function $H: \mathbb{B}_{p}^{n} \rightarrow \mathbb{R}$ is given by $H(x)=h\left(\|x\|_{p}\right)$ with

$$
h(r)=\frac{1}{p^{n / p} \Gamma\left(1+\frac{n}{p}\right)} \frac{1}{\left(1-r^{p}\right)^{1+n / p}} \int_{0}^{\infty} s^{n / p} e^{-\frac{1}{p} s r^{p}\left(1-r^{p}\right)^{-1}} \mathbf{W}(\mathrm{d} s), \quad r \in[0,1] .
$$

In other words this means that

$$
\begin{aligned}
\int_{\mathbb{B}_{p}^{n}} f(x) \mathbf{P}_{\mathbf{W}, n, p}(\mathrm{~d} x) & =\mathbf{W}(\{0\}) \int_{\mathbb{S}_{p}^{n-1}} f(x) \mathbf{C}_{n, p}(\mathrm{~d} x)+\int_{\mathbb{B}_{p}^{n}} f(x) H(x) \mathbf{U}_{n, p}(\mathrm{~d} x) \\
& =\mathbf{W}(\{0\}) \int_{\mathbb{S}_{p}^{n-1}} f(x) \mathbf{C}_{n, p}(\mathrm{~d} x)+\int_{\mathbb{B}_{p}^{n}} f(x) h\left(\|x\|_{p}\right) \mathbf{U}_{n, p}(\mathrm{~d} x)
\end{aligned}
$$


for all non-negative measurable functions $f: \mathbb{B}_{p}^{n} \rightarrow \mathbb{R}$. The class of measures of the form $\mathbf{P}_{\mathbf{W}, n, p}$ contains the following important cases, which are of particular interest (see Theorem 1, Theorem 3, Corollary 3, and Corollary 4 in [5]):

(i) If $\mathbf{W}$ is the exponential distribution with rate $1 / p$ (and mean $p$ ), then $\mathbf{W}(\{0\})=0, H \equiv 1$, and $\mathbf{P}_{\mathbf{W}, n, p}$ reduces to the uniform distribution $\mathbf{U}_{n, p}$ on $\mathbb{B}_{p}^{n}$.

(ii) If $\mathbf{W}=\delta_{0}$ is the Dirac measure concentrated at 0 , then $\mathbf{W}(\{0\})=1, H \equiv 0$, and $\mathbf{P}_{\mathbf{W}, n, p}$ is just the cone probability measure on $\mathbb{B}_{p}^{n}$.

(iii) If $\mathbf{W}=\operatorname{Gamma}(\alpha, 1 / p)$ is a gamma distribution with shape parameter $\alpha>0$ and rate $1 / p$, then $\mathbf{P}_{\mathbf{W}, n, p}$ is the beta-type probability measure on $\mathbb{B}_{p}^{n}$ with Lebesgue density given by

$$
x \mapsto \frac{\Gamma\left(\alpha+\frac{n}{p}\right)}{\Gamma(\alpha)\left(2 \Gamma\left(1+\frac{1}{p}\right)\right)^{n}}\left(1-\|x\|_{p}^{p}\right)^{\alpha-1}, \quad x \in \mathbb{B}_{p}^{n} .
$$

In particular, if $\alpha=m / p$ for some $m \in \mathbb{N}$, this is the image of the cone probability measure $\mathbf{C}_{n+m, p}$ on $\mathbb{B}_{p}^{n+m}$ under the orthogonal projection onto the first $n$ coordinates. Similarly, if $\alpha=1+m / p$, this distribution arises as the image of the uniform distribution $\mathbf{U}_{n+m, p}$ on $\mathbb{B}_{p}^{n+m}$ under the same orthogonal projection.

2.3. Gaussian and $p$-generalized Gaussian random variables. Let us denote, for $1 \leq p<\infty$, by $\left(Z_{i}\right)_{i \in \mathbb{N}}$ a sequence of independent copies of a $p$-generalized Gaussian random variable with Lebesgue density

$$
f_{p}(x)=\frac{e^{-\frac{|x|^{p}}{p}}}{2 p^{1 / p} \Gamma(1+1 / p)}, \quad x \in \mathbb{R} .
$$

Defining

$$
M_{p}(q):=\frac{p^{q / p}}{q+1} \frac{\Gamma\left(1+\frac{q+1}{p}\right)}{\Gamma(1+1 / p)}
$$

for $q \geq 1$, we can express the moments of $p$-generalized Gaussian random variables as follows:

$$
\mathbb{E}\left|Z_{1}\right|^{q}=M_{p}(q) \quad \text { and } \quad \operatorname{Cov}\left(\left|Z_{1}\right|^{r},\left|Z_{1}\right|^{s}\right)=M_{p}(r+s)-M_{p}(r) M_{p}(s),
$$

where $q, r, s \geq 1$, see [3, Lemma 3.1]. In particular, $\operatorname{Var}\left|Z_{1}\right|^{q}=M_{p}(2 q)-M_{p}(q)^{2}$. We shall use these relations frequently in the proof of Theorem $\mathrm{C}$ and its general version Theorem 5.1.

If $p=2$, then a $p$-generalized Gaussian random variable is nothing else than a standard Gaussian random variable. In this text we shall denote a sequence of such independent random variables by $\left(g_{i}\right)_{i \in \mathbb{N}}$.

2.4. Background material from large deviations theory. Let $(\Omega, \mathcal{A}, \mathbb{P})$ be a probability space and $\left(X_{n}\right)_{n \in \mathbb{N}}$ be a sequence of random vectors taking values in $\mathbb{R}^{d}$ for some $d \geq 1$. Further, let $\left(s_{n}\right)_{n \in \mathbb{N}}$ be an increasing sequence of real numbers and $I: \mathbb{R}^{d} \rightarrow[0, \infty]$ be a lower semi-continuous function with compact level sets $\left\{x \in \mathbb{R}^{d}: I(x) \leq \alpha\right\}, \alpha \in \mathbb{R}$. One says that $\left(X_{n}\right)_{n \in \mathbb{N}}$ satisfies a large deviations principle (LDP) on $\mathbb{R}^{d}$ with speed $s_{n}$ and rate function $I$ provided that

$$
-\inf _{x \in A^{\circ}} I(x) \leq \liminf _{n \rightarrow \infty} s_{n}^{-1} \log \mathbb{P}\left[X_{n} \in A\right] \leq \limsup _{n \rightarrow \infty} s_{n}^{-1} \log \mathbb{P}\left[X_{n} \in A\right] \leq-\inf _{x \in \bar{A}} I(x)
$$

for all Borel measurable $A \subseteq \mathbb{R}^{d}$. Especially, if $A$ is an $I$-continuity set, that is, if $A$ satisfies $I\left(A^{\circ}\right)=I(\bar{A})$ with $I(A):=\inf \{I(x): x \in A\}$, one has the exact limit relation

$$
\lim _{n \rightarrow \infty} s_{n}^{-1} \log \mathbb{P}\left[X_{n} \in A\right]=-I(A) .
$$

Let $d_{1}, d_{2} \in \mathbb{N}$ and suppose that $\left(X_{n}\right)_{n \in \mathbb{N}}$ is a sequence of $\mathbb{R}^{d_{1}}$-valued random vectors and that $\left(Y_{n}\right)_{n \in \mathbb{N}}$ is a sequence of $\mathbb{R}^{d_{2}}$-random vectors. We assume that both sequences satisfy LDPs with the same speed. The next result, taken from [2, Proposition 2.4], yields that also the sequence of $\mathbb{R}^{d_{1}+d_{2}}$-valued random vectors $\left(X_{n}, Y_{n}\right)$ satisfies an LDP and provides the form of the rate function.

Lemma 2.1 (LDP for independent vectors). Assume that $\left(X_{n}\right)_{n \in \mathbb{N}}$ satisfies an LDP on $\mathbb{R}^{d_{1}}$ with speed $s_{n}$ and rate function $I_{X}$ and that $\left(Y_{n}\right)_{n \in \mathbb{N}}$ satisfies an $L D P$ on $\mathbb{R}^{d_{2}}$ with speed $s_{n}$ and rate function $I_{Y}$. Then, if $X_{n}$ and $Y_{n}$ are independent for each $n \in \mathbb{N}$, the sequence of random vectors $\left(X_{n}, Y_{n}\right)$ satisfies an LDP on $\mathbb{R}^{d_{1}+d_{2}}$ with speed $s_{n}$ and rate function $I$ given by $I(x):=I_{X}\left(x_{1}\right)+I_{Y}\left(x_{2}\right), x=\left(x_{1}, x_{2}\right) \in \mathbb{R}^{d_{1}} \times \mathbb{R}^{d_{2}}$. 
Next, assume that a sequence $\left(X_{n}\right)_{n \in \mathbb{N}}$ of random variables satisfies an LDP with speed $s_{n}$ and rate function $I$. Suppose now that $\left(Y_{n}\right)_{n \in \mathbb{N}}$ is a sequence of random variables that are 'close' to the ones from the first sequence. The next result provides conditions under which in such a situation an LDP from the first can be transferred to the second sequence, see [11, Theorem 4.2.13] or [25, Lemma 27.13].

Lemma 2.2 (Exponential equivalence). Let $\left(X_{n}\right)_{n \in \mathbb{N}}$ and $\left(Y_{n}\right)_{n \in \mathbb{N}}$ be two sequence of $\mathbb{R}^{d}$-valued random vectors and assume that $\left(X_{n}\right)_{n \in \mathbb{N}}$ satisfies an $L D P$ on $\mathbb{R}^{d}$ with speed $s_{n}$ and rate function $I$. Further, suppose that the two sequences $\left(X_{n}\right)_{n \in \mathbb{N}}$ and $\left(Y_{n}\right)_{n \in \mathbb{N}}$ are exponentially equivalent, i.e.,

$$
\limsup _{n \rightarrow \infty} s_{n}^{-1} \log \mathbb{P}\left[\left\|X_{n}-Y_{n}\right\|_{2}>\delta\right]=-\infty
$$

for any $\delta>0$. Then $\left(Y_{n}\right)_{n \in \mathbb{N}}$ satisfies an LDP on $\mathbb{R}^{d}$ with the same speed and the same rate function.

Let us now recall what is known as Cramér's theorem for sequences of real-valued random variables. It provides an LDP for sequences of independent and identically distributed random variables, see [11, Theorem 2.2.3]. The rate function in Cramér's theorem is identified as the Legendre-Fenchel transform of the cumulant generating function of the involved random variables.

Lemma 2.3 (Cramér's theorem). Let $\left(X_{n}\right)_{n \in \mathbb{N}}$ be a sequence of independent and identically distributed random variables. Assume that $\mathbb{E}\left[e^{\lambda X_{1}}\right]<\infty$ for some $\lambda>0$. Then the sequence of random variables $\frac{1}{n} \sum_{i=1}^{n} X_{i}$ satisfies an LDP on $\mathbb{R}$ with speed $n$ and rate function $I(x)=\sup \left\{\lambda x-\log \mathbb{E}\left[e^{\lambda X_{1}}\right]: \lambda \in \mathbb{R}\right\}$.

Finally, we consider the possibility to transport a large deviations principle to another one by means of a a sequence of functions. We recall the following version of the contraction principle from [11, Corollary 4.2.21].

Lemma 2.4 (Contraction principle). Let $d_{1}, d_{2} \in \mathbb{N}$ and let $F: \mathbb{R}^{d_{1}} \rightarrow \mathbb{R}^{d_{2}}$ be a continuous function. Suppose that $\left(X_{n}\right)_{n \in \mathbb{N}}$ is a sequence of $\mathbb{R}^{d_{1}}$-valued random variables that satisfies an LDP on $\mathbb{R}^{d_{1}}$ with speed $s_{n}$ and rate function $I$. Further, suppose that for each $n \in \mathbb{N}, F_{n}: \mathbb{R}^{d_{1}} \rightarrow \mathbb{R}^{d_{2}}$ is a measurable function such that for all $\delta>0$,

$$
\limsup _{n \rightarrow \infty} s_{n}^{-1} \log \mathbb{P}\left[X_{n} \in \Gamma_{n, \delta}\right]=-\infty,
$$

where $\Gamma_{n, \delta}:=\left\{x \in \mathbb{R}^{d_{1}}:\left\|F_{n}(x)-F(x)\right\|_{2}>\delta\right\}$. Then the sequence of $\mathbb{R}^{d_{2}}$-valued random variables $\left(F_{n}\left(X_{n}\right)\right)_{n \in \mathbb{N}}$ satisfies an LDP on $\mathbb{R}^{d_{2}}$ with the same speed and with rate function $I \circ F^{-1}$.

2.5. Moderate deviations in $\mathbb{R}^{d}$. A moderate deviations principle (MDP) is formally nothing else than a large deviations principle. As already explained in the introduction, the difference is that LDPs provide estimates on the scale of a law of large numbers, while MDPs typically describe the probabilities at scales between a law of large numbers and a central limit theorem. An important tool for us will be the following MDP for sums of independent and identically distributed random vectors, see [11, Theorem 3.7.1].

Lemma 2.5 (MDP for sums of i.i.d. random vectors). Let $\left(X_{n}\right)_{n \in \mathbb{N}}$ be a sequence of independent and identically distributed random vectors in $\mathbb{R}^{d}$ and let $\left(t_{n}\right)_{n \in \mathbb{N}}$ be sequence of positive real numbers such that $t_{n}=\omega(1)$ and $t_{n}=o(\sqrt{n})$. We assume that $X_{1}$ is centered, its covariance matrix $\mathbf{C}=\operatorname{Cov}\left(X_{1}\right)$ is invertible, and $\log \mathbb{E}\left[e^{\left\langle\lambda, X_{1}\right\rangle}\right]<\infty$ for all $\lambda$ in a ball around the origin having positive radius. Then the sequence of random vectors $\frac{1}{t_{n} \sqrt{n}} \sum_{i=1}^{n} X_{i}, n \in \mathbb{N}$, satisfies an $L D P$ (i.e., an MDP as the sum is scaled by $t_{n} \sqrt{n}$ ) with speed $t_{n}^{2}$ and rate function $I(x)=\frac{1}{2}\left\langle x, \mathbf{C}^{-1} x\right\rangle, x \in \mathbb{R}^{d}$.

\section{Proof of Theorem A}

Let us recall from [1, Theorem 1.15] (see also [17] and [31]) that if the KLS conjecture were true, there would exist an absolute constant $C \in(0, \infty)$ such that, for every $n \in \mathbb{N}$ and all $t>0$,

$$
\mathbb{P}\left[\left|\frac{\left\|\xi_{n}\right\|_{2}}{\sqrt{k_{n}}}-1\right|>t\right] \leq 2 e^{-C t \sqrt{k_{n}}}
$$

Therefore, we have

and

$$
\log \mathbb{P}\left[\frac{\left\|\xi_{n}\right\|_{2}}{\sqrt{k_{n}}}-1>t\right] \leq \log \mathbb{P}\left[\left|\frac{\left\|\xi_{n}\right\|_{2}}{\sqrt{k_{n}}}-1\right|>t\right] \leq \log 2-C t \sqrt{k_{n}}
$$

$$
\log \mathbb{P}\left[\frac{\left\|\xi_{n}\right\|_{2}}{\sqrt{k_{n}}}<1-t\right] \leq \log \mathbb{P}\left[\left|\frac{\left\|\xi_{n}\right\|_{2}}{\sqrt{k_{n}}}-1\right|>t\right] \leq \log 2-C t \sqrt{k_{n}}
$$


and thus, for every $n \in \mathbb{N}$ and $t>0$,

$$
\frac{\log \mathbb{P}\left[\frac{\left\|\xi_{n}\right\|_{2}}{\sqrt{k_{n}}}>1+t\right]}{s_{n}} \leq \frac{\log 2}{s_{n}}-\frac{C t \sqrt{k_{n}}}{s_{n}}
$$

and

$$
\frac{\log \mathbb{P}\left[\frac{\left\|\xi_{n}\right\|_{2}}{\sqrt{k_{n}}}<1-t\right]}{s_{n}} \leq \frac{\log 2}{s_{n}}-\frac{C t \sqrt{k_{n}}}{s_{n}} .
$$

Therefore, taking the limit inferior, as $n \rightarrow \infty$, and taking into account the assumption that the sequence of random variables $\frac{\left\|X_{n}\right\|_{2}}{\sqrt{k_{n}}}$ satisfies (1) with speed $s_{n}$ and rate function $\mathbb{I}$, we make the following observation: if $s_{n}=o\left(\sqrt{k_{n}}\right)$, then for every $t>0$,

$$
-\inf _{x \in(1+t, \infty)} \mathbb{I}(x) \leq-\infty \quad \text { and } \quad-\inf _{x \in(-\infty, 1-t)} \mathbb{I}(x) \leq-\infty
$$

which implies that $\mathbb{I}$ is identically equal to $+\infty$ on $\mathbb{R} \backslash\{1\}$. Since

$$
\mathbb{P}\left[\frac{\left\|\xi_{n}\right\|_{2}}{\sqrt{k_{n}}} \in \mathbb{R}\right]=1
$$

we have that, for every $n \in \mathbb{N}$,

$$
\frac{\log \mathbb{P}\left[\frac{\left\|\xi_{n}\right\|_{2}}{\sqrt{k_{n}}} \in \mathbb{R}\right]}{s_{n}}=0 .
$$

Hence, taking the limit as $n \rightarrow \infty$,

$$
0=-\inf _{x \in \mathbb{R}} \mathbb{I}(x)=-\mathbb{I}(1),
$$

which implies that $\mathbb{I}$ would coincide with the singular rate function $\mathbb{I}_{0}$, a contradiction to our assumption that $\mathbb{I} \neq \mathbb{I}_{0}$.

If otherwise $s_{n} \approx \sqrt{k_{n}}$, then for any $t>0$ there exists a constant $C_{1} \in(0, \infty)$ such that

$$
-\inf _{x \in(t+1, \infty)} \mathbb{I}(x) \leq-C_{1} t
$$

which implies that

$$
\frac{\inf _{x \in(t+1, \infty)} \mathbb{I}(x)}{t+1}>C_{1} \frac{t}{t+1}
$$

for all $t>0$. This is equivalent to the fact that, for every $t>1$,

$$
\frac{\inf _{x \in(t, \infty)} \mathbb{I}(x)}{t}>C_{1} \frac{t-1}{t} .
$$

Now, for every $t_{0}>1$ we have that for all $t \in\left[t_{0}, \infty\right), C_{1} \frac{t-1}{t}>C_{2}\left(t_{0}\right)$. Thus, for any $t_{0}>1$,

$$
\inf _{t>t_{0}} \frac{\inf _{x \in(t, \infty)} \mathbb{I}(x)}{t} \geq C_{2}\left(t_{0}\right) \text {. }
$$

However, this is a contradiction to our assumption.

\section{Proof of Theorem B and its generalization}

In this section we prove the following generalized version of Theorem B. For this recall the definition of the probability measures $\mathbf{P}_{\mathbf{W}, n, p}$ on $\mathbb{B}_{p}^{n}$.

Theorem 4.1 (MDP on the critical scale, general version). Let $1 \leq p<\infty$ and $\left(k_{n}\right)_{n \in \mathbb{N}}$ be a sequence of positive integers such that $1 \leq k_{n} \leq n, k_{n}=\omega(1)$, and $k_{n}=o(n)$. Also let $\left(\mathbf{W}_{n}\right)_{n \in \mathbb{N}}$ be a sequence of probability measures on $[0, \infty)$ and for each $n \in \mathbb{N}$ let $X_{n}$ be a random vector with distribution $\mathbf{P}_{\mathbf{W}_{n}, n, p}$. Independently of $X_{n}$, let $E_{n}$ be a uniformly distributed $k_{n}$-dimensional random subspace for each $n \in \mathbb{N}$, and define $\mathscr{Z}_{n, p}:=n^{1 / p}\left\|P_{E_{n}} X_{n}\right\|_{2}$. Suppose that the sequence of random variables $W_{n}$ with distribution $\mathbf{W}_{n}$ satisfies an LDP with speed $n$ and a rate function $\mathbb{I}_{W}$ satisfying $\mathbb{I}_{W}(x) \neq 0$ for all $x \neq 0$. Then the same conclusions as in Theorem B hold. 
4.1. Probabilistic representation. The proof of Theorem 4.1 is based on a suitable probabilistic representation of the random variables $\mathscr{Z}_{n, p}$. For the case that the random vectors $X_{n}$ are uniformly distributed on $\mathbb{B}_{p}^{n}$ such a representation was derived in [2, Lemma 3.1] and for the general situation considered here it is the content of [3, Proposition 2.7]. It says that

$$
k_{n}^{-1 / 2} \mathscr{Z}_{n, p}=\frac{n^{1 / p}}{k_{n}^{1 / 2}}\left\|P_{E_{n}} X_{n}\right\|_{2} \stackrel{d}{=} \frac{n^{1 / p}}{k_{n}^{1 / 2}} \frac{\left(\sum_{i=1}^{k_{n}} g_{i}^{2}\right)^{1 / 2}}{\left(\sum_{i=1}^{n} g_{i}^{2}\right)^{1 / 2}} \frac{\left(\sum_{i=1}^{n}\left|Z_{i}\right|^{2}\right)^{1 / 2}}{\left(\sum_{i=1}^{n}\left|Z_{i}\right|^{p}+W_{n}\right)^{1 / p}},
$$

where $g_{1}, \ldots, g_{n}$ are independent standard Gaussian random variables and $Z_{1}, \ldots, Z_{n}$ are independent $p$ generalized Gaussian random variables, which are also independent of all $g_{i}$ 's. Thus,

$$
\frac{n^{1 / p}}{k_{n}^{1 / 2}}\left\|P_{E_{n}} X_{n}\right\|_{2} \stackrel{d}{=} \frac{\left(\frac{1}{k_{n}} \sum_{i=1}^{k_{n}} g_{i}^{2}\right)^{1 / 2}}{\left(\frac{1}{n} \sum_{i=1}^{n} g_{i}^{2}\right)^{1 / 2}} \frac{\left(\frac{1}{n} \sum_{i=1}^{n}\left|Z_{i}\right|^{2}\right)^{1 / 2}}{\left(\frac{1}{n} \sum_{i=1}^{n}\left|Z_{i}\right|^{p}+\frac{W_{n}}{n}\right)^{1 / p}} .
$$

4.2. Proof of Theorem 4.1 for $2 \leq p<\infty$. As a consequence of Cramér's theorem (Lemma 2.3) the sequence of random variables $\frac{1}{k_{n}} \sum_{i=1}^{k_{n}} g_{i}^{2}$ satisfies an LDP with speed $k_{n}$ and rate function

$$
\mathbb{I}_{G}(x)= \begin{cases}\frac{x-1}{2}-\frac{1}{2} \log x & : x>0 \\ +\infty & : \text { otherwise }\end{cases}
$$

compare with [2, Lemma 5.4]. Consequently, by the contraction principle (Lemma 2.4) the sequence of random variables $\left(\frac{M_{p}(2)}{k_{n}} \sum_{i=1}^{k_{n}} g_{i}^{2}\right)^{1 / 2}$ satisfies an LDP with the same speed and rate function

$$
\mathbb{I}_{1}(x)=\mathbb{I}_{G}\left(\frac{x^{2}}{M_{p}(2)}\right)= \begin{cases}\frac{\frac{x^{2}}{M_{p}(2)}-1}{2}-\log \frac{x}{\sqrt{M_{p}(2)}} & : x>0 \\ +\infty & : \text { otherwise }\end{cases}
$$

which is the rate function from the statement of the theorem. In the same way, the sequence $\left(\frac{1}{n} \sum_{i=1}^{n}\left|Z_{i}\right|^{p}\right)^{1 / p}$ satisfies an LDP with speed $n$ and rate function

$$
\mathbb{I}_{2}(x)= \begin{cases}\frac{x^{p}-1}{p}-\log x & : x>0 \\ +\infty & : \text { otherwise }\end{cases}
$$

The rate functions were explicitly given by [2, Lemma 5.4]. However, the authors realised that there was an overlooked misprint in the rate function $\mathbb{I}_{2}(x)$ given there, so we explicitly write the rate function here.

What remains to show is that the sequences of random variables $k_{n}^{-1 / 2} \mathscr{Z}_{n, p}$ and $\left(\frac{M_{p}(2)}{k_{n}} \sum_{i=1}^{k_{n}} g_{i}^{2}\right)^{1 / 2}$ are exponentially equivalent (recall Lemma 2.2). For this, we fix $\delta, \varepsilon>0$ and write

$$
\begin{gathered}
\mathbb{P}\left[\left(\frac{M_{p}(2)}{k_{n}} \sum_{i=1}^{k_{n}} g_{i}^{2}\right)^{1 / 2}\left|1-\frac{\left(\frac{1}{n} \sum_{i=1}^{n} \frac{Z_{i}^{2}}{M_{p}(2)}\right)^{1 / 2}}{\left(\frac{1}{n} \sum_{i=1}^{n} g_{i}^{2}\right)^{1 / 2}\left(\frac{1}{n} \sum_{i=1}^{n}\left|Z_{i}\right|^{p}+\frac{W_{n}}{n}\right)^{1 / p}}\right|>\delta\right] \\
\leq \mathbb{P}\left[\left(\frac{M_{p}(2)}{k_{n}} \sum_{i=1}^{k_{n}} g_{i}^{2}\right)^{1 / 2}>\frac{\delta}{\varepsilon}\right]+\mathbb{P}\left[1-\frac{\left(\frac{1}{n} \sum_{i=1}^{n} \frac{Z_{i}^{2}}{M_{p}(2)}\right)^{1 / 2}}{\left(\frac{1}{n} \sum_{i=1}^{n} g_{i}^{2}\right)^{1 / 2}\left(\frac{1}{n} \sum_{i=1}^{n}\left|Z_{i}\right|^{p}+\frac{W_{n}}{n}\right)^{1 / p}}>\varepsilon\right] \\
+\mathbb{P}\left[1-\frac{\left(\frac{1}{n} \sum_{i=1}^{n} \frac{Z_{i}^{2}}{M_{p}(2)}\right)^{1 / 2}}{\left(\frac{1}{n} \sum_{i=1}^{n} g_{i}^{2}\right)^{1 / 2}\left(\frac{1}{n} \sum_{i=1}^{n}\left|Z_{i}\right|^{p}+\frac{W_{n}}{n}\right)^{1 / p}}<-\varepsilon\right]=: T_{1}+T_{2}+T_{3} .
\end{gathered}
$$


We further analyse the term $T_{2}$ and observe that, for every $\varepsilon_{1}>0$,

$$
\begin{aligned}
T_{2} \leq \mathbb{P} & {\left[\left(\frac{1}{n} \sum_{i=1}^{n} \frac{Z_{i}^{2}}{M_{p}(2)}\right)^{1 / 2}<(1-\varepsilon)^{1 / 3}\right]+\mathbb{P}\left[\left(\frac{1}{n} \sum_{i=1}^{n} g_{i}^{2}\right)^{1 / 2}>(1-\varepsilon)^{-1 / 3}\right] } \\
& +\mathbb{P}\left[\frac{1}{n} \sum_{i=1}^{n}\left|Z_{i}\right|^{p}>(1-\varepsilon)^{-p / 3}-\varepsilon_{1}\right]+\mathbb{P}\left[\frac{W_{n}}{n}>\varepsilon_{1}\right] .
\end{aligned}
$$

By Cramér's theorem (see Lemma 2.3), the sequences of random variables $\frac{1}{n} \sum_{i=1}^{n} \frac{Z_{i}^{2}}{M_{p}(2)}, \frac{1}{n} \sum_{i=1}^{n} g_{i}^{2}$, and $\frac{1}{n} \sum_{i=1}^{n}\left|Z_{i}\right|^{p}$ satisfy LDPs with speed $n$ and rate functions only vanishing at 1 . Taking $\varepsilon_{1}$ such that (1$\varepsilon)^{-p / 3}-\varepsilon_{1}>1$ and taking into account that, by assumption also the sequence of random variables $W_{n} / n$ satisfies an LDP with speed $n$ and a rate function that does not vanish on $\left(\varepsilon_{1}, \infty\right)$ we have that the term $T_{2}$ decays exponentially with speed $n$ and hence satisfies

$$
\limsup _{n \rightarrow \infty} \frac{1}{k_{n}} \log T_{2}=-\infty
$$

since $k_{n}=o(n)$ by assumption. The same argument also yields that

which in turn leads to

$$
\limsup _{n \rightarrow \infty} \frac{1}{k_{n}} \log T_{3}=-\infty
$$

$$
\begin{aligned}
& \limsup _{n \rightarrow \infty} \frac{M_{p}(2)}{k_{n}} \log \mathbb{P}\left[\left(\frac{M_{p}(2)}{k_{n}} \sum_{i=1}^{k_{n}} g_{i}^{2}\right)^{1 / 2}\left|1-\frac{\left(\frac{1}{n} \sum_{i=1}^{n} \frac{Z_{i}^{2}}{M_{p}(2)}\right)^{1 / 2}}{\left(\frac{1}{n} \sum_{i=1}^{n} g_{i}^{2}\right)^{1 / 2}\left(\frac{1}{n} \sum_{i=1}^{n}\left|Z_{i}\right|^{p}+\frac{W_{n}}{n}\right)^{1 / p}}\right|>\delta\right] \\
& \leq \limsup _{n \rightarrow \infty} \frac{1}{k_{n}} \log T_{1}=-\mathbb{I}_{1}\left(\frac{\delta}{\varepsilon}\right)=-\frac{\frac{\delta^{2}}{\varepsilon^{2} M_{p}(2)}+1}{2}-\log \frac{\delta}{\varepsilon \sqrt{M_{p}(2)}}
\end{aligned}
$$

with $\mathbb{I}_{1}$ given by (7). As $\varepsilon \rightarrow 0$ the last expression tends to $-\infty$ for any $\delta>0$. This shows the desired exponential equivalence.

4.3. Proof of Theorem 4.1 for $1 \leq p<2$. The idea of the proof of Theorem 4.1 for $1 \leq p<2$ is similar to that for $2 \leq p<\infty$ : identify the dominating term(s) in the probabilistic representation (6) and show exponential equivalence of $k_{n}^{-1 / 2}\left\|\mathscr{Z}_{n, p}\right\|_{2}$ to them. However, the analysis in the case where $1 \leq p<2$ is a bit more subtle, since depending on the growth of $k_{n}$ relative to $n^{p / 2}$ different terms can take over the dominating role. Moreover, if $k_{n}=n^{p / 2}$ there is more than one such term.

4.3.1. The case $k_{n}=o\left(n^{p / 2}\right)$. This situation is similar to that where $2 \leq p<\infty$. But now the sequence of random variables $\left(\frac{1}{n} \sum_{i=1}^{n} Z_{i}^{2}\right)^{1 / 2}$ satisfies an LDP with speed $n^{p / 2}$ instead of $n$ (see [2, Equation (5)]). This slowdown of the speed is due to the fact that for $1 \leq p<2$ the squares of $p$-generalized Gaussian random variables display heavier tails and do not have finite exponential moments. However, since the subspace dimensions satisfy $k_{n}=o\left(n^{p / 2}\right)$, the proof still works in this situation.

4.3.2. The case $k_{n}=n^{p / 2}$. To deal with this case we start by observing that by Lemma 2.1 the sequence of random vectors

$$
\left(\left(\frac{1}{k_{n}} \sum_{i=1}^{k_{n}} g_{i}^{2}\right)^{1 / 2},\left(\frac{1}{n} \sum_{i=1}^{n} Z_{i}^{2}\right)^{1 / 2}\right)
$$

satisfies an LDP on $\mathbb{R}^{2}$ with speed $n^{p / 2}$ and rate function $\mathbb{I}_{G+Z}(x, y)=\mathbb{I}_{G}(x)+\mathbb{I}_{Z}(y)$. Here,

$$
\mathbb{I}_{G}(x)=\left\{\begin{array}{ll}
\frac{x^{2}-1}{2}-\log x & : x>0 \\
+\infty & : \text { otherwise }
\end{array} \quad \text { and } \quad \mathbb{I}_{Z}(y)= \begin{cases}\frac{1}{p}\left(y^{2}-m_{p}\right)^{p / 2} & : y>m_{p} \\
+\infty & : \text { otherwise }\end{cases}\right.
$$

where the constant $m_{p}$ is given in Theorem B and the precise form of the LDPs for the individual sequences follow from [2, Lemma 5.4 and Equation (5)]. By the contraction principle (Lemma 2.4) this implies that the sequence of random variables $\left(\frac{1}{k_{n}} \sum_{i=1}^{k_{n}} g_{i}^{2}\right)^{1 / 2} \cdot\left(\frac{1}{n} \sum_{i=1}^{n} Z_{i}^{2}\right)^{1 / 2}$ satisfies an LDP with speed $n^{p / 2}$ and rate function $\mathbb{I}$ as given in the statement of Theorem B (b2). It remains to prove that the sequences of random 
variables $\left(\frac{1}{k_{n}} \sum_{i=1}^{k_{n}} g_{i}^{2}\right)^{1 / 2} \cdot\left(\frac{1}{n} \sum_{i=1}^{n} Z_{i}^{2}\right)^{1 / 2}$ and $n^{-p / 2}\left\|\mathscr{Z}_{n, p}\right\|$ are exponentially equivalent (recall Lemma 2.2). To show this, fix $\delta, \varepsilon>0$ and observe that

$$
\begin{gathered}
\mathbb{P}\left[\left(\frac{1}{k_{n}} \sum_{i=1}^{k_{n}} g_{i}^{2}\right)^{1 / 2}\left(\frac{1}{n} \sum_{i=1}^{n} Z_{i}^{2}\right)^{1 / 2}\left|1-\frac{1}{\left(\frac{1}{n} \sum_{i=1}^{n} g_{i}^{2}\right)^{1 / 2}\left(\frac{1}{n} \sum_{i=1}^{n}\left|Z_{i}\right|^{p}+\frac{W_{n}}{n}\right)^{1 / p}}\right|>\delta\right] \\
\leq \mathbb{P}\left[\left(\frac{1}{k_{n}} \sum_{i=1}^{k_{n}} g_{i}^{2}\right)^{1 / 2}\left(\frac{1}{n} \sum_{i=1}^{n} Z_{i}^{2}\right)^{1 / 2}>\frac{\delta}{\varepsilon}\right]+\mathbb{P}\left[1-\frac{1}{\left(\frac{1}{n} \sum_{i=1}^{n} g_{i}^{2}\right)^{1 / 2}\left(\frac{1}{n} \sum_{i=1}^{n}\left|Z_{i}\right| p+\frac{W_{n}}{n}\right)^{1 / p}}>\varepsilon\right] \\
+\mathbb{P}\left[1-\frac{1}{\left(\frac{1}{n} \sum_{i=1}^{n} g_{i}^{2}\right)^{1 / 2}\left(\frac{1}{n} \sum_{i=1}^{n}\left|Z_{i}\right|^{p}+\frac{W_{n}}{n}\right)^{1 / p}}<-\varepsilon\right]=: T_{1}+T_{2}+T_{3} .
\end{gathered}
$$

Now, for $T_{2}$ we obtain that, for any $\varepsilon_{1}>0$,

$$
T_{2} \leq \mathbb{P}\left[\left(\frac{1}{n} \sum_{i=1}^{n} g_{i}^{2}\right)^{1 / 2}>(1-\varepsilon)^{-1 / 2}\right]+\mathbb{P}\left[\frac{1}{n} \sum_{i=1}^{n}\left|Z_{i}\right|^{p}>(1-\varepsilon)^{-p / 2}-\varepsilon_{1}\right]+\mathbb{P}\left[\frac{W_{n}}{n}>\varepsilon_{1}\right] .
$$

By Cramér's theorem (Lemma 2.3), the sequences of random variables $\frac{1}{n} \sum_{i=1}^{n} g_{i}^{2}$ and $\frac{1}{n} \sum_{i=1}^{n}\left|Z_{i}\right|^{p}$ satisfy an LDP with speed $n$, and, by assumption, $W_{n} / n$ also satisfies an LDP with speed $n$. Moreover, taking $\varepsilon_{1}$ small enough so that $(1-\varepsilon)^{-p / 2}-\varepsilon_{1}>1$, the corresponding rate functions do not vanish on $\left((1-\varepsilon)^{-1 / 2}, \infty\right)$, $\left((1-\varepsilon)^{-p / 2}-\varepsilon_{1}, \infty\right)$, and $\left(\varepsilon_{1}, \infty\right)$. Since a similar argument applies to $T_{3}$, we conclude that

$$
\limsup _{n \rightarrow \infty} \frac{1}{n^{p / 2}} \log T_{2} \leq-\infty \quad \text { and } \quad \limsup _{n \rightarrow \infty} \frac{1}{n^{p / 2}} \log T_{3} \leq-\infty .
$$

Thus,

$$
\begin{aligned}
& \limsup _{n \rightarrow \infty} \frac{1}{n^{p / 2}} \log \mathbb{P}\left[\left(\frac{1}{k_{n}} \sum_{i=1}^{k_{n}} g_{i}^{2}\right)^{1 / 2}\left(\frac{1}{n} \sum_{i=1}^{n} Z_{i}^{2}\right)^{1 / 2}\left|1-\frac{1}{\left(\frac{1}{n} \sum_{i=1}^{n} g_{i}^{2}\right)^{1 / 2}\left(\frac{1}{n} \sum_{i=1}^{n}\left|Z_{i}\right|^{p}+\frac{W_{n}}{n}\right)^{1 / p}}\right|>\delta\right] \\
& \leq \limsup _{n \rightarrow \infty} \frac{1}{n^{p / 2}} \log T_{1}=-\mathbb{I}\left(\frac{\delta}{\varepsilon}\right),
\end{aligned}
$$

where $\mathbb{I}$ is the rate function from the statement of Theorem B (b2). Taking into account the explicit form of $\mathbb{I}$, we conclude that $\mathbb{I}(\delta / \varepsilon) \rightarrow \infty$, as $\varepsilon \rightarrow 0$. This proves the result for the case that $k_{n}=n^{p / 2}$.

4.3.3. The case $k_{n}=\omega\left(n^{p / 2}\right)$. In this case we claim that the sequence of random variables $\left(\frac{1}{n} \sum_{i=1}^{n} Z_{i}^{2}\right)^{1 / 2}$ is exponentially equivalent to $k_{n}^{-1 / 2} \mathscr{Z}_{n, p}$. In fact, for $\delta, \varepsilon>0$ we can write

$$
\begin{gathered}
\mathbb{P}\left[\left(\frac{1}{n} \sum_{i=1}^{n} Z_{i}^{2}\right)^{1 / 2}\left|1-\frac{\left(\frac{1}{k_{n}} \sum_{i=1}^{k_{n}} g_{i}^{2}\right)^{1 / 2}}{\left(\frac{1}{n} \sum_{i=1}^{n} g_{i}^{2}\right)^{1 / 2}\left(\frac{1}{n} \sum_{i=1}^{n}\left|Z_{i}\right|^{p}+\frac{W_{n}}{n}\right)^{1 / p}}\right|>\delta\right] \\
\leq \mathbb{P}\left[\left(\frac{1}{n} \sum_{i=1}^{n} Z_{i}^{2}\right)^{1 / 2}>\frac{\delta}{\varepsilon}\right]+\mathbb{P}\left[1-\frac{\left(\frac{1}{k_{n}} \sum_{i=1}^{k_{n}} g_{i}^{2}\right)^{1 / 2}}{\left(\frac{1}{n} \sum_{i=1}^{n} g_{i}^{2}\right)^{1 / 2}\left(\frac{1}{n} \sum_{i=1}^{n}\left|Z_{i}\right|^{p}+\frac{W_{n}}{n}\right)^{1 / p}}>\varepsilon\right] \\
+\mathbb{P}\left[1-\frac{\left(\frac{1}{k_{n}} \sum_{i=1}^{k_{n}} g_{i}^{2}\right)^{1 / 2}}{\left(\frac{1}{n} \sum_{i=1}^{n} g_{i}^{2}\right)^{1 / 2}\left(\frac{1}{n} \sum_{i=1}^{n}\left|Z_{i}\right|^{p}+\frac{W_{n}}{n}\right)^{1 / p}}<-\varepsilon\right]=: T_{1}+T_{2}+T_{3},
\end{gathered}
$$


and for $T_{2}$, we obtain that, for any $\varepsilon_{1}>0$,

$$
\begin{aligned}
T_{2} \leq \mathbb{P} & {\left[\frac{1}{k_{n}} \sum_{i=1}^{k_{n}} g_{i}^{2}<(1-\varepsilon)^{2 / 3}\right]+\mathbb{P}\left[\frac{1}{n} \sum_{i=1}^{n} g_{i}^{2}>(1-\varepsilon)^{-2 / 3}\right] } \\
& +\mathbb{P}\left[\frac{1}{n} \sum_{i=1}^{n}\left|Z_{i}\right|^{p}>(1-\varepsilon)^{-p / 3}-\varepsilon_{1}\right]+\mathbb{P}\left[\frac{W_{n}}{n}>\varepsilon_{1}\right] .
\end{aligned}
$$

Once again, by Cramér's theorem (Lemma 2.3), the sequences of random variables $\frac{1}{n} \sum_{i=1}^{n} g_{i}^{2}$ and $\frac{1}{n} \sum_{i=1}^{n}\left|Z_{i}\right|^{p}$ satisfy an LDP with speed $n$, and, by assumption, $W_{n} / n$ also satisfies an LDP with speed $n$. Again by Cramér's theorem (Lemma 2.3), the sequence of random variables $\frac{1}{k_{n}} \sum_{i=1}^{k_{n}} g_{i}^{2}$ satisfies an LDP with speed $k_{n}$. Also and as already discussed above, taking $\varepsilon_{1}$ small enough so that $(1-\varepsilon)^{-p / 3}-\varepsilon_{1}>1$, the corresponding rate functions do not vanish on $\left(-\infty,(1-\varepsilon)^{2 / 3},\left((1-\varepsilon)^{-2 / 3}, \infty\right),\left((1-\varepsilon)^{-p / 3}-\varepsilon_{1}, \infty\right)\right.$, and $\left(\varepsilon_{1}, \infty\right)$. This proves that for suitable constants $c_{1}, c_{2}>0$,

$$
\limsup _{n \rightarrow \infty} \frac{1}{n^{p / 2}} \log T_{2} \leq-\limsup _{n \rightarrow \infty}\left(\frac{c_{1} k_{n}}{n^{p / 2}}+\frac{c_{2} n}{n^{p / 2}}\right)=-\infty,
$$

since $k_{n}=\omega\left(n^{p / 2}\right)$ by assumption and $1 \leq p<2$. Similarly, one has that

$$
\limsup _{n \rightarrow \infty} \frac{1}{n^{p / 2}} \log T_{3}=-\infty
$$

and hence

$$
\begin{aligned}
& \limsup _{n \rightarrow \infty} \frac{1}{n^{p / 2}} \log \mathbb{P}\left[\left(\frac{1}{n} \sum_{i=1}^{n} Z_{i}^{2}\right)^{1 / 2}\left|1-\frac{\left(\frac{1}{k_{n}} \sum_{i=1}^{k_{n}} g_{i}^{2}\right)^{1 / 2}}{\left(\frac{1}{n} \sum_{i=1}^{n} g_{i}^{2}\right)^{1 / 2}\left(\frac{1}{n} \sum_{i=1}^{n}\left|Z_{i}\right|^{p}+\frac{W_{n}}{n}\right)^{1 / p}}\right|>\delta\right] \\
& \leq \limsup _{n \rightarrow \infty} \frac{1}{n^{p / 2}} \log T_{1}=-\mathbb{I}\left(\frac{\delta}{\varepsilon}\right),
\end{aligned}
$$

where $\mathbb{I}$ is the rate function of the LDP for the sequence of random variables $\left(\frac{1}{n} \sum_{i=1}^{n} Z_{i}^{2}\right)^{1 / 2}$, which holds at speed $n^{p / 2}$. We have that

$$
\mathbb{I}(x)= \begin{cases}\frac{1}{p}\left(x^{2}-m_{p}\right)^{p / 2} & : x>\sqrt{m_{p}} \\ +\infty & : \text { otherwise }\end{cases}
$$

with the constant $m_{p}$ as in Theorem B according to [2, Equation (5)]. In particular, this shows that $\mathbb{I}(\delta / \varepsilon) \rightarrow$ $\infty$, as $\varepsilon \rightarrow 0$, and proves the exponential equivalence of the sequences of random variables $\left(\frac{1}{n} \sum_{i=1}^{n} Z_{i}^{2}\right)^{1 / 2}$ and $k_{n}^{-1 / 2} \mathscr{Z}_{n, p}$. The proof of Theorem 4.1 is thus complete.

\section{Proof of Theorem C and its generalization}

Recall the notions and notation introduced before Theorem $\mathrm{C}$ and recall the definition of the probability measures $\mathbf{P}_{\mathbf{W}, n, p}$ on $\mathbb{B}_{p}^{n}$. The goal of this section is to prove the following result, which contains Theorem $\mathrm{C}$ as a special case.

Theorem 5.1 (MDP on subcritical scales, general version). Let $2 \leq p<\infty$ and $\left(k_{n}\right)_{n \in \mathbb{N}}$ be a sequence of integers such that $1 \leq k_{n} \leq n$ and $\lim _{n \rightarrow \infty} \frac{k_{n}}{n}=\lambda \in[0,1]$. Assume that $k_{n}=\omega(1)$, and that the sequence $\left(t_{n}\right)_{n \in \mathbb{N}}$ of positive real numbers satisfies $t_{n}=\omega(1), t_{n}=o\left(\sqrt{k_{n}}\right)$, and either $t_{n}=o\left(\sqrt{n-k_{n}}\right)$ or $\left(n-k_{n}\right)=o\left(\frac{\sqrt{k_{n}}}{t_{n}}\right)$. For each $n \in \mathbb{N}$ let $\mathbf{W}_{n}$ be a probability measure on $\mathbb{R}$ such that

$$
\lim _{n \rightarrow \infty} t_{n}^{-2} \log \mathbf{W}_{n}\left(\left(\delta t_{n} \sqrt{n}, \infty\right)\right)=-\infty
$$

for every $\delta>0$. Further, let $X_{n}$ be a random point with distribution $\mathbf{P}_{\mathbf{W}_{n}, n, p}$ and $E_{n}$ be a uniformly distributed $k_{n}$-dimensional random subspace of $\mathbb{R}^{n}$. Assume independence of $X_{n}$ and $E_{n}$ and put

$$
\mathscr{X}_{n, p}:=n^{1 / p} \sqrt{\frac{\Gamma\left(\frac{1}{p}\right)}{p^{2 / p} \Gamma\left(\frac{3}{p}\right)}}\left\|P_{E_{n}} X_{n}\right\|_{2}-\sqrt{k_{n}} .
$$


Then the sequence of random variables $t_{n}^{-1} \mathscr{X}_{n, p}$ satisfies an $M D P$ on $\mathbb{R}$ with speed $t_{n}^{2}$ and rate function $\mathbb{I}: \mathbb{R} \rightarrow[0, \infty), \mathbb{I}(x)=\alpha_{p, \lambda} x^{2}$ with $\alpha_{p, \lambda}$ given by $(2)$.

5.1. Probabilistic representation. As for the proof of the central limit theorem in [3] and the large deviations principle in [2], a suitable probabilistic representation for the target random variable $t_{n}^{-1} \mathscr{X}_{n, p}$ in terms of families of independent random variables turns out to be one of the most crucial ingredients also in our proof of the moderate deviations principle. In the context of the MDP, it is in fact the representation used in the proof of the central limit theorem, which is most suitable and which we develop further in the present text. To ease comparison we adopt the notation from [3] and recall from the proof of [3, Theorem 1.1] that for each $n \in \mathbb{N}$, we have

$$
\begin{array}{r}
\frac{\mathscr{X}_{n, p}}{t_{n}} \stackrel{d}{=} \frac{\sqrt{\lambda_{n}}}{2 M_{p}(2)} \xi_{p, 2}^{(n)}-\frac{\sqrt{\lambda_{n}}}{p} \xi_{p, p}^{(n)}+\frac{1-\lambda_{n}}{2} \zeta_{1}^{(n)}-\frac{\sqrt{\lambda_{n}\left(1-\lambda_{n}\right)}}{2} \zeta_{2}^{(n)}-\sqrt{\lambda_{n}} \frac{W_{n}}{p t_{n} \sqrt{n}} \\
+\frac{\sqrt{\lambda_{n}}}{t_{n} \sqrt{n}} \Psi_{p}\left(\frac{t_{n} \xi_{p, 2}^{(n)}}{\sqrt{n}}, \frac{t_{n} \xi_{p, p}^{(n)}}{\sqrt{n}}, \frac{t_{n} \zeta_{1}^{(n)}}{\sqrt{k_{n}}}, \frac{t_{n} \zeta_{3}^{(n)}}{\sqrt{n}}, \frac{W_{n}}{n}\right),
\end{array}
$$

where the random variable $W_{n}$ has distribution $\mathbf{W}_{n}$,

$$
\begin{array}{rlrl}
\xi_{p, 2}^{(n)} & :=\frac{1}{t_{n} \sqrt{n}} \sum_{i=1}^{n}\left(Z_{i}^{2}-M_{p}(2)\right), & \xi_{p, p}^{(n)}:=\frac{1}{t_{n} \sqrt{n}} \sum_{i=1}^{n}\left(\left|Z_{i}\right|^{p}-1\right), \\
\zeta_{1}^{(n)}:=\frac{1}{t_{n} \sqrt{k_{n}}} \sum_{i=1}^{k_{n}}\left(g_{i}^{2}-1\right), & \zeta_{2}^{(n)}:=\frac{1}{t_{n} \sqrt{n-k_{n}}} \sum_{i=k_{n}+1}^{n}\left(g_{i}^{2}-1\right), \\
\zeta_{3}^{(n)}:=\sqrt{\lambda_{n}} \zeta_{1}^{(n)}+\sqrt{1-\lambda_{n}} \zeta_{2}^{(n)}=\frac{1}{t_{n} \sqrt{n}} \sum_{i=1}^{n}\left(g_{i}^{2}-1\right), &
\end{array}
$$

and where $\Psi_{p}: \mathbb{R}^{5} \rightarrow \mathbb{R}$ is a function with the property that there are two constants $M, \delta \in(0, \infty)$ such that $\left|\Psi_{p}(\mathbf{x})\right| \leq M\|\mathbf{x}\|_{2}^{2}$ whenever $\|\mathbf{x}\|_{2}<\delta$. At this point we shall remind the reader of our convention that $\left(Z_{i}\right)_{i \in \mathbb{N}}$ stands for a sequence of independent $p$-generalized Gaussian random variables and $\left(g_{i}\right)_{i \in \mathbb{N}}$ for a sequence of independent standard Gaussian random variables and that both sequences are assumed to be independent of each other. In addition, it is understood that the random variables $W_{n}$ are also independent of all $g_{i}$ 's and $Z_{i}$ 's. We also emphasize that a notation involving the symbol $\xi$ refers to random variables involving the $p$-generalized Gaussian random variables $Z_{i}$ only, while the symbol $\zeta$ refers to random elements only based on the standard Gaussians $g_{i}$.

Finally, we shall denote by $\mathscr{Y}_{n, p}$ the random variable given by

$$
\mathscr{Y}_{n, p}:=\frac{\sqrt{\lambda_{n}}}{2 M_{p}(2)} \xi_{p, 2}^{(n)}-\frac{\sqrt{\lambda_{n}}}{p} \xi_{p, p}^{(n)}+\frac{1-\lambda_{n}}{2} \zeta_{1}^{(n)}-\frac{\sqrt{\lambda_{n}\left(1-\lambda_{n}\right)}}{2} \zeta_{2}^{(n)}, \quad n \in \mathbb{N} .
$$

Moreover, we define

$$
\widetilde{\mathscr{Y}}_{n, p}:=\frac{\sqrt{\lambda_{n}}}{2 M_{p}(2)} \xi_{p, 2}^{(n)}-\frac{\sqrt{\lambda_{n}}}{p} \xi_{p, p}^{(n)}+\frac{1-\lambda_{n}}{2} \zeta_{1}^{(n)}, \quad n \in \mathbb{N} .
$$

5.2. MDP for $p$-generalized Gaussian random variables. Specializing Lemma 2.5 for $d=1$ to normalized sums of $p$-generalized Gaussian random variables leads to the following MDP.

Lemma 5.2 (MDP for sums of powers of $p$-generalized Gaussians). Let $2 \leq q \leq p<\infty$ and $\left(Z_{i}\right)_{i \in \mathbb{N}}$ be $a$ sequence of independent copies of a p-generalized Gaussian random variable. Let $\left(t_{n}\right)_{n \in \mathbb{N}}$ be a sequence of positive real numbers such that $t_{n}=\omega(1)$ and $t_{n}=o(\sqrt{n})$. Then the sequence of random variables

$$
\xi_{p, q}^{(n)}:=\frac{1}{t_{n} \sqrt{n}} \sum_{i=1}^{n}\left(\left|Z_{i}\right|^{q}-M_{p}(q)\right), \quad n \in \mathbb{N},
$$

satisfies an $M D P$ on $\mathbb{R}$ with speed $t_{n}^{2}$ and rate function

$$
\mathscr{I}: \mathbb{R} \rightarrow[0, \infty), \quad \mathscr{I}(x)=\frac{x^{2}}{2 \operatorname{Var}\left[\left|Z_{1}\right|^{q}\right]}=\frac{x^{2}}{2\left(M_{p}(2 q)-M_{p}(q)^{2}\right)} .
$$

Proof. Clearly, $\mathbb{E}\left[\left|Z_{1}\right|^{q}-M_{p}(q)\right]=M_{p}(q)-M_{p}(q)=0$ and, using the notation of Lemma $2.5, \mathbf{C}^{-1}=$ $1 / \operatorname{Var}\left[\left|Z_{1}\right|^{q}\right]$ (in fact, $\mathbf{C}$ is a $(1 \times 1)$-matrix in our case). In addition, we have that $\operatorname{Var}\left[\left|Z_{1}\right|^{q}\right]=M_{p}(2 q)-M_{p}(q)^{2}$ from (5). The result is now a direct consequence of Lemma 2.5, since exponential moments exists by our assumption that $2 \leq q \leq p$. 
Remark 5.3. It is not strictly necessary to assume that $2 \leq q$ in the previous lemma. However, we imposed this condition as we will apply the result for $q=2$, and it shows the reason why we need the condition $p \geq 2$ in Theorem 5.1 .

5.3. MDP for Gaussian random variables. As a consequence of Lemma 5.2 obtained in the previous section, applied with $p=q=2$, we obtain the following MDP for sums of random vectors composed by Gaussian random variables.

Corollary 5.4 (MDP for sums of Gaussians). Let $\left(g_{i}\right)_{i \in \mathbb{N}}$ be a sequence of standard Gaussian random variables. Then we have that if $k_{n}=\omega(1)$, and $\left(t_{n}\right)_{n \in \mathbb{N}}$ is a sequence of positive real numbers such that $t_{n}=\omega(1)$ and $t_{n}=o\left(\sqrt{k_{n}}\right)$, the sequence of random variables $\zeta_{1}^{(n)}:=\frac{1}{t_{n} \sqrt{k_{n}}} \sum_{i=1}^{k_{n}}\left(g_{i}^{2}-1\right)$ follows an $M D P$ on $\mathbb{R}$ with speed $t_{n}^{2}$ and rate function $\mathscr{I}(x)=\frac{x^{2}}{4}, x \in \mathbb{R}$ and, if $n-k_{n}=\omega(1)$ and $\left(t_{n}\right)_{n \in \mathbb{N}}$ is a sequence of positive real numbers such that $t_{n}=\omega(1)$ and $t_{n}=o\left(\sqrt{n-k_{n}}\right)$, the sequence of random variables $\zeta_{2}^{(n)}:=\frac{1}{t_{n} \sqrt{n-k_{n}}} \sum_{i=k_{n}+1}^{n}\left(g_{i}^{2}-1\right)$ follows an MDP on $\mathbb{R}$ with speed $t_{n}^{2}$ and rate function $\mathscr{I}(x)=\frac{x^{2}}{4}, x \in \mathbb{R}$. Consequently, if $k_{n}=\omega(1), n-k_{n}=\omega(1), t_{n}=\omega(1), t_{n}=o\left(\sqrt{k_{n}}\right)$, and $t_{n}=o\left(\sqrt{n-k_{n}}\right)$, the sequence of random vectors $\zeta^{(n)}:=\left(\zeta_{1}^{(n)}, \zeta_{2}^{(n)}\right)$ satisfies an $M D P$ on $\mathbb{R}^{2}$ with speed $t_{n}^{2}$ and rate function $\mathscr{I}(x)=\frac{\|x\|_{2}^{2}}{4}$, $x \in \mathbb{R}^{2}$.

5.4. MDP for correlated vectors of $p$-generalized Gaussian random variables. In a next step we prove an MDP for a sum of random vectors, whose entries are correlated and composed of $p$-generalized Gaussian random variables.

Lemma 5.5 (Bivariate MDP for $p$-generalized Gaussians). Let $2 \leq p<\infty$ and $\left(Z_{i}\right)_{i \in \mathbb{N}}$ be a sequence of independent copies of a p-generalized Gaussian random variable. Assume that $\left(t_{n}\right)_{n \in \mathbb{N}}$ is a sequence of positive real numbers such that $t_{n}=\omega(1)$ and $t_{n}=o(\sqrt{n})$. Then the sequence of random vectors in $\mathbb{R}^{2}$ given by

$$
\left(\zeta_{1}^{(n)}, \zeta_{2}^{(n)}\right)=\frac{1}{t_{n} \sqrt{n}} \sum_{i=1}^{n}\left(Z_{i}^{2}-M_{p}(2),\left|Z_{i}\right|^{p}-1\right)
$$

satisfies an MDP on $\mathbb{R}^{2}$ with speed $t_{n}^{2}$ and rate function

$$
J\left(x_{1}, x_{2}\right)=\frac{1}{2 A_{p}}\left(p x_{1}^{2}+p^{4 / p}\left(\frac{\Gamma\left(\frac{5}{p}\right)}{\Gamma\left(\frac{1}{p}\right)}-\frac{\Gamma\left(\frac{3}{p}\right)^{2}}{\Gamma\left(\frac{1}{p}\right)^{2}}\right) x_{2}^{2}-4 p^{2 / p} \frac{\Gamma\left(\frac{3}{p}\right)}{\Gamma\left(\frac{1}{p}\right)} x_{1} x_{2}\right), \quad\left(x_{1}, x_{2}\right) \in \mathbb{R}^{2},
$$

with the constant $A_{p}$ given by

$$
A_{p}:=p^{4 / p}\left(\frac{\Gamma\left(\frac{5}{p}\right)}{\Gamma\left(1+\frac{1}{p}\right)}-(p+4) \frac{\Gamma\left(\frac{3}{p}\right)^{2}}{\Gamma\left(\frac{1}{p}\right)^{2}}\right) .
$$

Proof. We start by noting that the random vector $\left(\left|Z_{1}\right|^{2}-M_{p}(2),\left|Z_{1}\right|^{p}-1\right)$ is centered. Its $2 \times 2$ covariance matrix $\mathbf{C}$ is given by

$$
\mathbf{C}=\left(\begin{array}{ll}
c_{11} & c_{12} \\
c_{12} & c_{22}
\end{array}\right)
$$

where

$$
\begin{aligned}
& c_{11}=\operatorname{Cov}\left(\left|Z_{1}\right|^{2}-M_{p}(2),\left|Z_{1}\right|^{2}-M_{p}(2)\right)=\operatorname{Var}\left|Z_{1}\right|^{2}=M_{p}(4)-M_{p}(2)^{2}, \\
& c_{12}=\operatorname{Cov}\left(\left|Z_{1}\right|^{2}-M_{p}(2),\left|Z_{1}\right|^{p}-1\right)=M_{p}(p+2)-M_{p}(2) M_{p}(p)=M_{p}(p+2)-M_{p}(2), \\
& c_{22}=\operatorname{Cov}\left(\left|Z_{1}\right|^{p}-1,\left|Z_{1}\right|^{p}-1\right)=M_{p}(2 p)-M_{p}(p)^{2}=p,
\end{aligned}
$$

recall (5). Consequently, $\operatorname{det} \mathbf{C}=p\left(M_{p}(4)-M_{p}(2)^{2}\right)-\left(M_{p}(p+2)-M_{p}(2)\right)^{2}$ and

$$
\mathbf{C}^{-1}=\frac{1}{\operatorname{det} \mathbf{C}}\left(\begin{array}{cc}
p & M_{p}(2)-M_{p}(p+2) \\
M_{p}(2)-M_{p}(p+2) & M_{p}(4)-M_{p}(2)^{2}
\end{array}\right)
$$


Expressing this in terms of gamma functions, using (4), we arrive at $\operatorname{det} \mathbf{C}=A_{p}$ and

$$
\mathbf{C}^{-1}=\frac{1}{A_{p}}\left(\begin{array}{cc}
p & -2 p^{2 / p} \frac{\Gamma\left(\frac{3}{p}\right)}{\Gamma\left(\frac{1}{p}\right)} \\
-2 p^{2 / p} \frac{\Gamma\left(\frac{3}{p}\right)}{\Gamma\left(\frac{1}{p}\right)} & p^{4 / p}\left(\frac{\Gamma\left(\frac{5}{p}\right)}{\Gamma\left(\frac{1}{p}\right)}-\frac{\Gamma\left(\frac{3}{p}\right)^{2}}{\Gamma\left(\frac{1}{p}\right)^{2}}\right)
\end{array}\right) .
$$

Since by assumption $p \geq 2$, exponential moments exist and we can apply Lemma 2.5. Thus, we conclude that the sequence of pairs $\left(\left(\zeta_{1}^{(n)}, \zeta_{2}^{(n)}\right)\right)_{n \in \mathbb{N}}$ satisfies an MDP on $\mathbb{R}^{2}$ with speed $t_{n}^{2}$ and rate function

$$
J\left(x_{1}, x_{2}\right)=\frac{1}{2}\left\langle\left(x_{1}, x_{2}\right)^{T}, \mathbf{C}^{-1}\left(x_{1}, x_{2}\right)^{T}\right\rangle, \quad\left(x_{1}, x_{2}\right) \in \mathbb{R}^{2} .
$$

Using the explicit form of $\mathbf{C}^{-1}$, the result follows.

5.5. Intermediate MDP. Recall the definition of the random variables $\xi_{p, 2}^{(n)}, \xi_{p, p}^{(n)}, \zeta_{1}^{(n)}$, and $\zeta_{2}^{(n)}$. Using what has been proved in the previous section, we arrive at the following MDP for the sequence of random vectors $\left(\xi_{p, 2}^{(n)}, \xi_{p, p}^{(n)}, \zeta_{1}^{(n)}\right)$ and $\left(\xi_{p, 2}^{(n)}, \xi_{p, p}^{(n)}, \zeta_{1}^{(n)}, \zeta_{2}^{(n)}\right)$.

Lemma 5.6 (Multivariate MDP for the core term). The following sequences of random vectors satisfy MDPs with speed $t_{n}^{2}$ and the given rate functions:

(a) If $k_{n}=\omega(1), t_{n}=\omega(1)$, and $t_{n}=o\left(\sqrt{k_{n}}\right)$, then the sequence of random vectors $\left(\xi_{p, 2}^{(n)}, \xi_{p, p}^{(n)}, \zeta_{1}^{(n)}\right)$ satisfies an MDP on $\mathbb{R}^{3}$ with speed $t_{n}^{2}$ and rate function

$$
I\left(x_{1}, x_{2}, x_{3}\right)=J\left(x_{1}, x_{2}\right)+\frac{x_{3}^{2}}{4},
$$

where $J(\cdot, \cdot)$ is the rate function from Lemma 5.5 .

(b) If $k_{n}=\omega(1), n-k_{n}=\omega(1), t_{n}=\omega(1), t_{n}=o\left(\sqrt{k_{n}}\right)$, and $t_{n}=o\left(\sqrt{n-k_{n}}\right)$, then the sequence of random vectors $\left(\xi_{p, 2}^{(n)}, \xi_{p, p}^{(n)}, \zeta_{1}^{(n)}, \zeta_{2}^{(n)}\right)$ satisfies an $M D P$ on $\mathbb{R}^{4}$ with speed $t_{n}^{2}$ and rate function

$$
I\left(x_{1}, x_{2}, x_{3}, x_{4}\right)=J\left(x_{1}, x_{2}\right)+\frac{x_{3}^{2}+x_{4}^{2}}{4},
$$

where $J(\cdot, \cdot)$ is the rate function from Lemma 5.5 .

Proof. We only prove part (b), since the proof of part (a) is completely analogous and simpler. We note that $\xi_{p, 2}^{(n)}$ and $\xi_{p, p}^{(n)}$ are independent from $\zeta_{1}^{(n)}$ and $\zeta_{2}^{(n)}$. We have seen in Lemma 5.5 that the sequence of random vectors $\left(\xi_{p, 2}^{(n)}, \xi_{p, p}^{(n)}\right)$ satisfies an MDP on $\mathbb{R}^{2}$ with speed $t_{n}^{2}$ and rate function $J\left(x_{1}, x_{2}\right)$. Also, Corollary 5.4 implies that the sequence of random vectors $\left(\zeta_{1}^{(n)}, \zeta_{2}^{(n)}\right)$ satisfies an MDP on $\mathbb{R}^{2}$ with speed $t_{n}^{2}$ and rate function $L\left(x_{3}, x_{4}\right)=\frac{x_{3}^{2}+x_{4}^{2}}{4}$. Thus, the sequence of random vectors $\left(\xi_{p, 2}^{(n)}, \xi_{p, p}^{(n)}, \zeta_{1}^{(n)}, \zeta_{2}^{(n)}\right)$ satisfies an MDP on $\mathbb{R}^{4}$ with the same speed $t_{n}^{2}$, whose rate function is the sum of the rate functions $J\left(x_{1}, x_{2}\right)$ and $L\left(x_{3}, x_{4}\right)$, see Lemma 2.1 .

Next, we shall apply the contraction principle from Lemma 2.4 to deduce the following intermediate MDP for the random sequence $\left(\mathscr{Y}_{n, p}\right)_{n \in \mathbb{N}}$.

Lemma 5.7 (Intermediate MDP for the core term). The following sequences of random variables satisfy $M D P s$ with speed $t_{n}^{2}$ and the given rate functions:

(a) If $k_{n}=\omega(1), t_{n}=\omega(1)$, and $t_{n}=o\left(\sqrt{k_{n}}\right)$, then the sequence of random variables $\left(\widetilde{\mathscr{Y}}_{n, p}\right)_{n \in \mathbb{N}}$ satisfies an $M D P$ on $\mathbb{R}$ with speed $t_{n}^{2}$ and rate function

$$
I(y)=\inf \left\{J\left(x_{1}, x_{2}\right)+\frac{x_{3}^{2}}{4}: \widetilde{F}\left(x_{1}, x_{2}, x_{3}\right)=y\right\}, \quad y \in \mathbb{R},
$$

with the function $\widetilde{F}: \mathbb{R}^{3} \rightarrow \mathbb{R}$ given by

$$
F\left(x_{1}, x_{2}, x_{3}\right):=\frac{\sqrt{\lambda}}{2 M_{p}(2)} x_{1}-\frac{\sqrt{\lambda}}{p} x_{2}+\frac{1-\lambda}{2} x_{3} .
$$


(b) If $k_{n}=\omega(1), n-k_{n}=\omega(1), t_{n}=\omega(1), t_{n}=o\left(\sqrt{k_{n}}\right)$, and $t_{n}=o\left(\sqrt{n-k_{n}}\right)$, then the sequence of random variables $\left(\mathscr{Y}_{n, p}\right)_{n \in \mathbb{N}}$ satisfies an MDP on $\mathbb{R}$ with speed $t_{n}^{2}$ and rate function

$$
I(y)=\inf \left\{J\left(x_{1}, x_{2}\right)+\frac{x_{3}^{2}+x_{4}^{2}}{4}: F\left(x_{1}, x_{2}, x_{3}, x_{4}\right)=y\right\}, \quad y \in \mathbb{R},
$$

with the function $F: \mathbb{R}^{4} \rightarrow \mathbb{R}$ given by

$$
F\left(x_{1}, x_{2}, x_{3}, x_{4}\right):=\frac{\sqrt{\lambda}}{2 M_{p}(2)} x_{1}-\frac{\sqrt{\lambda}}{p} x_{2}+\frac{1-\lambda}{2} x_{3}-\frac{\sqrt{\lambda(1-\lambda)}}{2} x_{4} .
$$

Proof. Again, we only prove part (b), since the proof of part (a) is completely analogous and simpler. For each $n \in \mathbb{N}$ let $F_{n}: \mathbb{R}^{4} \rightarrow \mathbb{R}$ be the function

$$
F_{n}\left(x_{1}, x_{2}, x_{3}, x_{4}\right):=\frac{\sqrt{\lambda_{n}}}{2 M_{p}(2)} x_{1}-\frac{\sqrt{\lambda_{n}}}{p} x_{2}+\frac{1-\lambda_{n}}{2} x_{3}-\frac{\sqrt{\lambda_{n}\left(1-\lambda_{n}\right)}}{2} x_{4}
$$

and observe that $F\left(x_{1}, x_{2}, x_{3}, x_{4}\right)$ is the pointwise limit of $F_{n}\left(x_{1}, x_{2}, x_{3}, x_{4}\right)$, as $n \rightarrow \infty$. Note that for each $n \in \mathbb{N}, \mathscr{Y}_{n, p}$ has the same distribution as $F_{n}\left(\xi_{p, 2}^{(n)}, \xi_{p, p}^{(n)}, \zeta_{1}^{(n)}, \zeta_{2}^{(n)}\right)$.

Our goal is to apply Lemma 2.4 to conclude from Lemma 5.6 the MDP for the random sequence $\left(\mathscr{Y}_{n, p}\right)_{n \in \mathbb{N}}$. For this we need to argue that

$$
\lim _{n \rightarrow \infty} \frac{1}{t_{n}^{2}} \log \mathbb{P}\left[\left(\xi_{p, 2}^{(n)}, \xi_{p, p}^{(n)}, \zeta_{1}^{(n)}, \zeta_{2}^{(n)}\right) \in \Gamma_{n, \delta}\right]=-\infty,
$$

where for $\delta>0, \Gamma_{n, \delta}$ is the set $\Gamma_{n, \delta}:=\left\{x \in \mathbb{R}^{4}:\left|F_{n}(x)-F(x)\right|>\delta\right\}$. To prove this, we first note that

$$
\begin{aligned}
\mathbb{P}\left[\left(\xi_{p, 2}^{(n)}, \xi_{p, p}^{(n)}, \zeta_{1}^{(n)}, \zeta_{2}^{(n)}\right) \in \Gamma_{n, \delta}\right] \leq & \mathbb{P}\left[\frac{\left|\sqrt{\lambda_{n}}-\sqrt{\lambda}\right|}{t_{n} \sqrt{n}}\left|\sum_{i=1}^{n}\left(Z_{i}^{2}-M_{p}(2)\right)\right|>\frac{\delta M_{p}(2)}{2}\right] \\
& +\mathbb{P}\left[\frac{\left|\sqrt{\lambda_{n}}-\sqrt{\lambda}\right|}{t_{n} \sqrt{n}}\left|\sum_{i=1}^{n}\left(\left|Z_{i}\right|^{p}-1\right)\right|>\frac{p \delta}{4}\right]+\mathbb{P}\left[\frac{\left|\lambda_{n}-\lambda\right|}{t_{n} \sqrt{k_{n}}}\left|\sum_{i=1}^{k_{n}}\left(g_{i}^{2}-1\right)\right|>\frac{\delta}{2}\right] \\
& +\mathbb{P}\left[\frac{\sqrt{\lambda_{n}\left(1-\lambda_{n}\right)}-\sqrt{\lambda(1-\lambda) \mid}}{t_{n} \sqrt{n-k_{n}}}\left|\sum_{i=k_{n}+1}^{n}\left(g_{i}^{2}-1\right)\right|>\frac{\delta}{2}\right] .
\end{aligned}
$$

If there exists a constant $c_{1}:=c_{1}(\delta, p) \in(0, \infty)$ such that $\frac{t_{n} \sqrt{n}}{\mid \sqrt{\lambda_{n}}-\sqrt{\lambda \mid}} \geq c_{1} n$, then, by Cramér's theorem in Lemma 2.3 the sequence $\frac{1}{n} \sum_{i=1}^{n}\left(Z_{i}^{2}-M_{p}(2)\right)$ satisfies an LDP with speed $n$ and some rate function. So, there exists a constant $c_{2}:=c_{2}(\delta, p) \in(0, \infty)$ only depending on $\delta$ and on $p$ such that

$$
\mathbb{P}\left[\frac{\left|\sqrt{\lambda_{n}}-\sqrt{\lambda}\right|}{t_{n} \sqrt{n}}\left|\sum_{i=1}^{n}\left(Z_{i}^{2}-M_{p}(2)\right)\right|>\frac{\delta M_{p}(2)}{2}\right] \leq \mathbb{P}\left[\frac{1}{n}\left|\sum_{i=1}^{n}\left(Z_{i}^{2}-M_{p}(2)\right)\right|>\frac{c_{1} \delta M_{p}(2)}{2}\right] \leq e^{-c_{2} n}
$$

so that

$$
\lim _{n \rightarrow \infty} \frac{1}{t_{n}^{2}} \log \mathbb{P}\left[\frac{\left|\sqrt{\lambda_{n}}-\sqrt{\lambda}\right|}{t_{n} \sqrt{n}}\left|\sum_{i=1}^{n}\left(Z_{i}^{2}-M_{p}(2)\right)\right|>\frac{\delta M_{p}(2)}{2}\right] \leq-\lim _{n \rightarrow \infty} \frac{c_{2} n}{t_{n}^{2}}=-\infty
$$

by our assumption on the growth of $t_{n}$. On the contrary, if such constant does not exist, then by Lemma 5.2 there exists another constant $c_{3}:=c_{3}(\delta, p) \in(0, \infty)$ depending on $\delta$ and $p$ only such that

$$
\mathbb{P}\left[\frac{\left|\sqrt{\lambda_{n}}-\sqrt{\lambda}\right|}{t_{n} \sqrt{n}}\left|\sum_{i=1}^{n}\left(Z_{i}^{2}-M_{p}(2)\right)\right|>\frac{\delta M_{p}(2)}{2}\right] \leq e^{-\frac{c_{3} t_{n}^{2}}{\left(\sqrt{\lambda_{n}}-\sqrt{\lambda}\right)^{2}}},
$$

which also implies that

$$
\lim _{n \rightarrow \infty} \frac{1}{t_{n}^{2}} \log \mathbb{P}\left[\frac{\left|\sqrt{\lambda_{n}}-\sqrt{\lambda}\right|}{t_{n} \sqrt{n}}\left|\sum_{i=1}^{n}\left(Z_{i}^{2}-M_{p}(2)\right)\right|>\frac{\delta M_{p}(2)}{2}\right] \leq-\lim _{n \rightarrow \infty} \frac{c_{3}}{\left(\sqrt{\lambda_{n}}-\sqrt{\lambda}\right)^{2}}=-\infty,
$$

since $\lambda_{n} \rightarrow \lambda$, as $n \rightarrow \infty$. Thus, in any case we have that

$$
\lim _{n \rightarrow \infty} \frac{1}{t_{n}^{2}} \log \mathbb{P}\left[\frac{\left|\sqrt{\lambda_{n}}-\sqrt{\lambda}\right|}{t_{n} \sqrt{n}}\left|\sum_{i=1}^{n} Z_{i}^{2}-M_{p}(2)\right|>\frac{\delta M_{p}(2)}{2}\right]=-\infty
$$


The rest of the terms are treated in the same way, which eventually proves (9).

5.6. Explicit form of the rate function. After having proved an intermediate MDP in Lemma 5.7, we shall now provide an explicit form for the rate function $I(y)$.

Lemma 5.8 (Explicit rate function). The rate functions $I: \mathbb{R} \rightarrow[0, \infty]$ in Lemma 5.7 (a) and (b) are given by $I(y)=\alpha_{p, \lambda} y^{2}$ with

$$
\alpha_{p, \lambda} \frac{2 \Gamma\left(\frac{1}{p}\right) \Gamma\left(\frac{3}{p}\right)^{2}}{(2 p-\lambda(4+3 p)) \Gamma\left(1+\frac{1}{p}\right) \Gamma\left(\frac{3}{p}\right)^{2}+\lambda \Gamma\left(\frac{1}{p}\right)^{2} \Gamma\left(\frac{5}{p}\right)} .
$$

Proof. For parameters $A, a, b, c \in \mathbb{R}$ let us introduce the function $\widetilde{G}: \mathbb{R}^{4} \rightarrow \mathbb{R}$ by

$$
\widetilde{G}\left(x_{1}, x_{2}, x_{3}, x_{4}\right):=\frac{1}{2 A}\left(a x_{1}^{2}+b x_{2}^{2}+c x_{1} x_{2}\right)+\frac{x_{3}^{2}+x_{4}^{2}}{4} .
$$

Our goal is to minimize this function subject to the condition that $\alpha x_{1}+\beta x_{2}+\gamma x_{3}+\delta x_{4}=y$ for fixed $\alpha, \beta, \gamma, \delta \in \mathbb{R}$ and given $y \in \mathbb{R}$. For that purpose we define $G: \mathbb{R}^{5} \rightarrow \mathbb{R}$ by

$$
G\left(x_{1}, x_{2}, x_{3}, x_{4}, \lambda\right):=\widetilde{G}\left(x_{1}, x_{2}, x_{3}, x_{4}\right)+\lambda\left(\alpha x_{1}+\beta x_{2}+\gamma x_{3}+\delta x_{4}-y\right) .
$$

Differentiation with respect to $x_{1}, \ldots, x_{4}$ gives

$$
\begin{aligned}
\frac{\partial G}{\partial x_{1}} & =\frac{1}{2 A}\left(2 a x_{1}+c x_{2}\right)+\alpha \lambda, & \frac{\partial G}{\partial x_{2}} & =\frac{1}{2 A}\left(2 b x_{2}+c x_{1}\right)+\beta \lambda, \\
\frac{\partial G}{\partial x_{3}} & =\frac{x_{3}}{2}+\gamma \lambda, & \frac{\partial G}{\partial x_{4}} & =\frac{x_{4}}{2}+\delta \lambda .
\end{aligned}
$$

The system $\frac{\partial G}{\partial x_{1}}=0, \frac{\partial G}{\partial x_{2}}=0, \frac{\partial G}{\partial x_{3}}=0, \frac{\partial G}{\partial x_{4}}=0$ has the unique solution

$$
x_{1}^{0}=\frac{2 A \lambda(\beta c-2 \alpha b)}{4 a b-c^{2}}, \quad x_{2}^{0}=\frac{2 A \lambda(\alpha c-2 \beta a)}{4 a b-c^{2}}, \quad x_{3}^{0}=-2 \gamma \lambda, \quad x_{4}^{0}=-2 \delta \lambda .
$$

Plugging this into $\frac{\partial G}{\partial \lambda}=\alpha x_{1}+\beta x_{2}+\gamma x_{3}+\delta x_{4}-y=0$ and solving for $\lambda$ yields the unique solution

$$
\lambda=y \frac{c^{2}-4 a b}{4 A\left(\alpha^{2} b+\beta^{2} a-\alpha \beta c\right)+2\left(4 a b-c^{2}\right)\left(\delta^{2}+\gamma^{2}\right)} .
$$

Plugging this in turn into the expressions for $x_{1}^{0}, x_{2}^{0}, x_{3}^{0}, x_{4}^{0}$ obtained above yields

$$
\begin{aligned}
& x_{1}^{0}=y \frac{A(2 \alpha b-\beta c)}{2 A\left(\alpha^{2} b+\beta^{2} a-\alpha \beta c\right)+2\left(4 a b-c^{2}\right)\left(\delta^{2}+\gamma^{2}\right)}, \\
& x_{2}^{0}=y \frac{A(2 \beta a-\alpha c)}{2 A\left(\alpha^{2} b+\beta^{2} a-\alpha \beta c\right)+2\left(4 a b-c^{2}\right)\left(\delta^{2}+\gamma^{2}\right)}, \\
& x_{3}^{0}=y \frac{\gamma\left(4 a b-c^{2}\right)}{2 A\left(\alpha^{2} b+\beta^{2} a-\alpha \beta c\right)+2\left(4 a b-c^{2}\right)\left(\delta^{2}+\gamma^{2}\right)}, \\
& x_{4}^{0}=y \frac{\delta\left(4 a b-c^{2}\right)}{2 A\left(\alpha^{2} b+\beta^{2} a-\alpha \beta c\right)+2\left(4 a b-c^{2}\right)\left(\delta^{2}+\gamma^{2}\right)} .
\end{aligned}
$$

Consequently,

$$
\widetilde{G}\left(x_{1}^{0}, x_{2}^{0}, x_{3}^{0}, x_{4}^{0}\right)=y^{2} \frac{4 a b-c^{2}}{4\left(2 A\left(\alpha^{2} b+\beta^{2} a-\alpha \beta c\right)+\left(4 a b-c^{2}\right)\left(\delta^{2}+\gamma^{2}\right)\right)} .
$$

In our set up, the parameters $A, a, b, c$ and $\alpha, \beta, \gamma, \delta$ are given as follows:

$$
A=A_{p}, \quad a=p, \quad b=p^{4 / p}\left(\frac{\Gamma\left(\frac{5}{p}\right)}{\Gamma\left(\frac{1}{p}\right)}-\frac{\Gamma\left(\frac{3}{p}\right)^{2}}{\Gamma\left(\frac{1}{p}\right)^{2}}\right), \quad c=-4 p^{2 / p} \frac{\Gamma\left(\frac{3}{p}\right)}{\Gamma\left(\frac{1}{p}\right)}
$$

and

$$
\alpha=\frac{\sqrt{\lambda}}{2 M_{p}(2)}, \quad \beta=\frac{\sqrt{\lambda}}{p}, \quad \gamma=\frac{1-\lambda}{2}, \quad \delta=\sqrt{\frac{\lambda(1-\lambda)}{2}} .
$$


Plugging this into the above expression leads to

$$
\frac{4 a b-c^{2}}{4\left(2 A\left(\alpha^{2} b+\beta^{2} a-\alpha \beta c\right)+\left(4 a b-c^{2}\right)\left(\delta^{2}+\gamma^{2}\right)\right)}=\frac{2 \Gamma\left(\frac{1}{p}\right) \Gamma\left(\frac{3}{p}\right)^{2}}{(2 p-\lambda(4+3 p)) \Gamma\left(1+\frac{1}{p}\right) \Gamma\left(\frac{3}{p}\right)^{2}+\lambda \Gamma\left(\frac{1}{p}\right)^{2} \Gamma\left(\frac{5}{p}\right)}=\alpha_{p, \lambda},
$$

after simplifications, where $\alpha_{p, \lambda}$ was defined in (2). Note that this covers both cases $\lim _{n \rightarrow \infty}\left(n-k_{n}\right)=\infty$ and $\lim _{n \rightarrow \infty}\left(n-k_{n}\right)<\infty$, since in the latter case we automatically have $\lambda=1$, which in turn corresponds to the choice $\gamma=\delta=0$. This completes the argument.

5.7. MDP for $\mathscr{X}_{n, p}$ via exponential equivalence. The purpose of this section is to complete the proof of the MDP for $\left(\mathscr{X}_{n, p}\right)_{n \in \mathbb{N}}$ in Theorem 5.1. We do this by showing that this sequence is exponentially equivalent to the random sequence $\left(\mathscr{Y}_{n, p}\right)_{n \in \mathbb{N}}$. Moreover, if $\left(n-k_{n}\right)=o\left(\frac{\sqrt{k_{n}}}{t_{n}}\right)$ we shall argue in addition that the random sequences $\left(\widetilde{\mathscr{Y}}_{n, p}\right)_{n \in \mathbb{N}}$ and $\left(\mathscr{Y}_{n, p}\right)_{n \in \mathbb{N}}$ are exponentially equivalent as well. As a consequence, $\left(\mathscr{X}_{n, p}\right)_{n \in \mathbb{N}}$ and $\left(\mathscr{Y}_{n, p}\right)_{n \in \mathbb{N}}$ satisfy an MDP at the same speed and with the same rate function (see Lemma 2.2), which implies that Theorem 5.1 follows from the intermediate MDP in Lemma 5.7. Moreover, the explicit form of the rate function is a consequence of Lemma 5.8. So, what remains to prove is the following exponential equivalence.

Lemma 5.9 (Exponential equivalence). If $k_{n}=\omega(1), t_{n}=\omega(1)$, and $t_{n}=o\left(\sqrt{k_{n}}\right)$ the sequence of random variables $t_{n}^{-1} \mathscr{X}_{n, p}$ and the sequence of random variables $\mathscr{Y}_{n, p}$ are exponentially equivalent. Moreover, if also $\left(n-k_{n}\right)=o\left(\frac{\sqrt{k_{n}}}{t_{n}}\right)$, then the sequences of random variables $\left(\widetilde{\mathscr{Y}}_{n, p}\right)_{n \in \mathbb{N}}$ and $\left(\mathscr{Y}_{n, p}\right)_{n \in \mathbb{N}}$ are exponentially equivalent.

Proof. We start by noting that, for any $\varepsilon>0$,

$$
\begin{aligned}
& \frac{1}{t_{n}^{2}} \log \mathbb{P}\left[\left|\frac{1}{t_{n}} \mathscr{X}_{n, p}-\mathscr{Y}_{n, p}\right|>\varepsilon\right] \\
& \leq \frac{1}{t_{n}^{2}} \log \left(\mathbb{P}\left[\frac{\sqrt{\lambda_{n}}}{p t_{n} \sqrt{n}}\left|W_{n}\right|>\frac{\epsilon}{2}\right]+\mathbb{P}\left[\frac{\sqrt{\lambda_{n}}}{t_{n} \sqrt{n}}\left|\Psi_{p}\left(\frac{t_{n} \xi_{p, 2}^{(n)}}{\sqrt{n}}, \frac{t_{n} \xi_{p, p}^{(n)}}{\sqrt{n}}, \frac{t_{n} \zeta_{1}^{(n)}}{\sqrt{k_{n}}}, \frac{t_{n} \zeta_{3}^{(n)}}{\sqrt{n}}, \frac{W_{n}}{n}\right)\right|>\frac{\epsilon}{2}\right]\right) \\
& \leq \frac{1}{t_{n}^{2}} \log \left(\mathbb{P}\left[\frac{\sqrt{\lambda_{n}}}{p t_{n} \sqrt{n}}\left|W_{n}\right|>\frac{\epsilon}{2}\right]+\mathbb{P}\left[\frac{\sqrt{\lambda_{n}}}{t_{n} \sqrt{n}} \mid\left(\frac{t_{n} \xi_{p, 2}^{(n)}}{\sqrt{n}}, \frac{t_{n} \xi_{p, p}^{(n)}}{\sqrt{n}}, \frac{t_{n} \zeta_{1}^{(n)}}{\sqrt{k_{n}}}, \frac{t_{n} \zeta_{3}^{(n)}}{\sqrt{n}}, \frac{W_{n}}{n}\right) \|_{2}^{2}>\frac{\varepsilon}{2 M}\right]\right) \\
& \left.+\mathbb{P}\left[\frac{\left|\Psi_{p}\left(\frac{t_{n} \xi_{p, 2}^{(n)}}{\sqrt{n}}, \frac{t_{n} \xi_{p, p}^{(n)}}{\sqrt{n}}, \frac{t_{n} \zeta_{1}^{(n)}}{\sqrt{k_{n}}}, \frac{t_{n} \zeta_{3}^{(n)}}{\sqrt{n}}, \frac{W_{n}}{n}\right)\right|}{\left\|\left(\frac{t_{n} \xi_{p, 2}^{(n)}}{\sqrt{n}}, \frac{t_{n} \xi_{p, p}^{(n)}}{\sqrt{n}}, \frac{t_{n} \zeta_{1}^{(n)}}{\sqrt{k_{n}}}, \frac{t_{n} \zeta_{3}^{(n)}}{\sqrt{n}}, \frac{W_{n}}{n}\right)\right\|_{2}^{2}}>M\right]\right)
\end{aligned}
$$

where $M$ is the constant associated to $\Psi_{p}$. On the one hand, by assumption (8), we have that

$$
\frac{1}{t_{n}^{2}} \log \mathbb{P}\left[\frac{\sqrt{\lambda_{n}}}{p t_{n} \sqrt{n}}\left|W_{n}\right|>\frac{\epsilon}{2}\right] \leq \frac{1}{t_{n}^{2}} \log \mathbb{P}\left[\left|W_{n}\right|>\frac{p \epsilon t_{n} \sqrt{n}}{2}\right] \rightarrow-\infty
$$

as $n \rightarrow \infty$. On the other hand, notice that, if $M$ and $\delta$ are the constants associated to $\Psi_{p}$,

$$
\begin{gathered}
\mathbb{P}\left[\frac{\left|\Psi_{p}\left(\frac{t_{n} \xi_{p, 2}^{(n)}}{\sqrt{n}}, \frac{t_{n} \xi_{p, p}^{(n)}}{\sqrt{n}}, \frac{t_{n} \zeta_{1}^{(n)}}{\sqrt{k_{n}}}, \frac{t_{n} \zeta_{3}^{(n)}}{\sqrt{n}}, \frac{W_{n}}{n}\right)\right|}{\left\|\left(\frac{t_{n} \xi_{p, 2}^{(n)}}{\sqrt{n}}, \frac{t_{n} \xi_{p, p}^{(n)}}{\sqrt{n}}, \frac{t_{n} \zeta_{1}^{(n)}}{\sqrt{k_{n}}}, \frac{t_{n} \zeta_{3}^{(n)}}{\sqrt{n}}, \frac{W_{n}}{n}\right)\right\|_{2}^{2}}>M\right] \\
\leq \mathbb{P}\left[\left\|\left(\frac{t_{n} \xi_{p, 2}^{(n)}}{\sqrt{n}}, \frac{t_{n} \xi_{p, p}^{(n)}}{\sqrt{n}}, \frac{t_{n} \zeta_{1}^{(n)}}{\sqrt{k_{n}}}, \frac{t_{n} \zeta_{3}^{(n)}}{\sqrt{n}}, \frac{W_{n}}{n}\right)\right\|_{2}>\delta\right] \\
+\mathbb{P}\left[\frac{t_{n}\left|\xi_{p, 2}^{(n)}\right|}{\sqrt{n}}>\frac{\delta}{\sqrt{5}}\right]+\mathbb{P}\left[\frac{t_{n}\left|\xi_{p, p}^{(n)}\right|}{\sqrt{n}}>\frac{\delta}{\sqrt{5}}\right]+\mathbb{P}\left[\frac{t_{n}\left|\zeta_{1}^{(n)}\right|}{\sqrt{k_{n}}}>\frac{\delta}{\sqrt{5}}\right] \\
+\mathbb{P}\left[\frac{t_{n}\left|\zeta_{3}^{(n)}\right|}{\sqrt{n}}>\frac{\delta}{\sqrt{5}}\right]+\mathbb{P}\left[\frac{\left|W_{n}\right|}{n}>\frac{\delta}{\sqrt{5}}\right] .
\end{gathered}
$$


By Cramér's theorem in Lemma 2.3, the sequence of random variables $\frac{t_{n}\left|\xi_{p, 2}^{(n)}\right|}{\sqrt{n}}=\frac{1}{n} \sum_{i=1}^{n}\left(Z_{i}^{2}-M_{p}(2)\right)$ follows an LDP with speed $n$. So, there exists a constant $c_{1}:=c_{1}(p, \delta) \in(0, \infty)$ depending on $\delta$ and $p$ only, and $N_{0} \in \mathbb{N}$ such that if $n \geq N_{0}$,

$$
\mathbb{P}\left[\frac{t_{n}\left|\xi_{p, 2}^{(n)}\right|}{\sqrt{n}}>\frac{\delta}{\sqrt{5}}\right] \leq e^{-c_{1} n}
$$

Therefore,

$$
\lim _{n \rightarrow \infty} \frac{1}{t_{n}^{2}} \log \mathbb{P}\left[\frac{t_{n}\left|\xi_{p, 2}^{(n)}\right|}{\sqrt{n}}>\frac{\delta}{\sqrt{5}}\right] \leq-\lim _{n \rightarrow \infty} \frac{c_{1} n}{t_{n}^{2}}=-\infty .
$$

In the same way we conclude that

$$
\lim _{n \rightarrow \infty} \frac{1}{t_{n}^{2}} \log \mathbb{P}\left[\frac{t_{n}\left|\xi_{p, p}^{(n)}\right|}{\sqrt{n}}>\frac{\delta}{\sqrt{5}}\right]-\infty \quad \text { and } \quad \lim _{n \rightarrow \infty} \frac{1}{t_{n}^{2}} \log \mathbb{P}\left[\frac{t_{n}\left|\zeta_{3}^{(n)}\right|}{\sqrt{n}}>\frac{\delta}{\sqrt{5}}\right]-\infty
$$

Also, the sequence of random variables $\frac{t_{n}\left|\zeta_{1}^{(n)}\right|}{\sqrt{k_{n}}}=\frac{1}{k_{n}} \sum_{i=1}^{k_{n}}\left(g_{i}^{2}-1\right)$ satisfies an LDP with speed $k_{n}$, implying that there exists a constant $c_{2}:=c_{2}(\delta) \in(0, \infty)$ only depending on $\delta$, and $N_{1} \in \mathbb{N}$ such that if $n \geq N_{1}$, then

$$
\mathbb{P}\left[\frac{t_{n}\left|\zeta_{1}^{(n)}\right|}{\sqrt{k_{n}}}>\frac{\delta}{\sqrt{5}}\right] \leq e^{-c_{2} k_{n}}
$$

Therefore, by our assumption on the growth of $k_{n}$, this implies that

$$
\lim _{n \rightarrow \infty} \frac{1}{t_{n}^{2}} \log \mathbb{P}\left[\frac{t_{n}\left|\zeta_{1}^{(n)}\right|}{\sqrt{k_{n}}}>\frac{\delta}{\sqrt{5}}\right] \leq-\lim _{n \rightarrow \infty} \frac{c_{2} k_{n}}{t_{n}^{2}}=-\infty
$$

Besides, by condition (8)

$$
\lim _{n \rightarrow \infty} \frac{1}{t_{n}^{2}} \log \mathbb{P}\left[\frac{\left|W_{n}\right|}{n}>\frac{\delta}{\sqrt{5}}\right] \leq \lim _{n \rightarrow \infty} \frac{1}{t_{n}^{2}} \log \mathbb{P}\left[\left|W_{n}\right|>\frac{\delta n}{\sqrt{5}}\right]=-\infty
$$

Finally,

$$
\begin{aligned}
& \mathbb{P}\left[\frac{\sqrt{\lambda_{n}}}{t_{n} \sqrt{n}}\right.\left.\left\|\left(\frac{t_{n} \xi_{p, 2}^{(n)}}{\sqrt{n}}, \frac{t_{n} \xi_{p, p}^{(n)}}{\sqrt{n}}, \frac{t_{n} \zeta_{1}^{(n)}}{\sqrt{k_{n}}}, \frac{t_{n} \zeta_{3}^{(n)}}{\sqrt{n}}, \frac{W_{n}}{n}\right)\right\|_{2}^{2}>\frac{\varepsilon}{2 M}\right] \\
& \leq \mathbb{P} {\left[\frac{t_{n} \xi_{p, 2}^{(n)}}{\sqrt{n}}>\frac{\sqrt{\varepsilon t_{n} \sqrt{n}}}{\lambda_{n}^{1 / 4} \sqrt{10 M}}\right]+\mathbb{P}\left[\frac{t_{n} \xi_{p, p}^{(n)}}{\sqrt{n}}>\frac{\sqrt{\varepsilon t_{n} \sqrt{n}}}{\lambda_{n}^{1 / 4} \sqrt{10 M}}\right] } \\
&+\mathbb{P}\left[\frac{t_{n} \zeta_{1}^{(n)}}{\sqrt{k_{n}}}>\frac{\sqrt{\varepsilon t_{n} \sqrt{n}}}{\lambda_{n}^{1 / 4} \sqrt{10 M}}\right]+\mathbb{P}\left[\frac{t_{n} \zeta_{3}^{(n)}}{\sqrt{n}}>\frac{\sqrt{\varepsilon t_{n} \sqrt{n}}}{\lambda_{n}^{1 / 4} \sqrt{10 M}}\right] \\
&+\mathbb{P}\left[\frac{\left|W_{n}\right|}{n}>\frac{\sqrt{\varepsilon t_{n} \sqrt{n}}}{\lambda_{n}^{1 / 4} \sqrt{10 M}}\right] .
\end{aligned}
$$

Like before, there exists a constant $c_{1}:=c_{1}(\varepsilon, M) \in(0, \infty)$ depending only on $\varepsilon$ and $M$, and $N_{0} \in \mathbb{N}$ such that, for all $n \geq N_{0}$,

$$
\mathbb{P}\left[\frac{t_{n} \xi_{p, 2}^{(n)}}{\sqrt{n}}>\frac{\sqrt{\varepsilon t_{n} \sqrt{n}}}{\lambda_{n}^{1 / 4} \sqrt{10 M}}\right] \leq \mathbb{P}\left[\frac{t_{n} \xi_{1}^{(n)}}{\sqrt{n}}>\frac{\sqrt{\varepsilon}}{\sqrt{10 M}}\right] \leq e^{-c_{1} n}
$$

which in turn implies that

$$
\lim _{n \rightarrow \infty} \frac{1}{t_{n}^{2}} \log \mathbb{P}\left[\frac{t_{n} \xi_{1}^{(n)}}{\sqrt{n}}>\frac{\sqrt{\varepsilon t_{n} \sqrt{n}}}{\lambda_{n}^{1 / 4} \sqrt{10 M}}\right] \leq-\lim _{n \rightarrow \infty} \frac{c_{1} n}{t_{n}^{2}}=-\infty .
$$


In the same way, we conclude that

$$
\begin{aligned}
& \lim _{n \rightarrow \infty} \frac{1}{t_{n}^{2}} \log \mathbb{P}\left[\frac{t_{n} \xi_{p, p}^{(n)}}{\sqrt{n}}>\frac{\sqrt{\varepsilon t_{n} \sqrt{n}}}{\lambda_{n}^{1 / 4} \sqrt{10 M}}\right]=-\infty, \\
& \lim _{n \rightarrow \infty} \frac{1}{t_{n}^{2}} \log \mathbb{P}\left[\frac{t_{n} \zeta_{3}^{(n)}}{\sqrt{n}}>\frac{\sqrt{\varepsilon t_{n} \sqrt{n}}}{\lambda_{n}^{1 / 4} \sqrt{10 M}}\right] \leq-\lim _{n \rightarrow \infty} \frac{c_{1} n}{t_{n}^{2}}=-\infty, \\
& \lim _{n \rightarrow \infty} \frac{1}{t_{n}^{2}} \log \mathbb{P}\left[\frac{t_{n} \zeta_{1}^{(n)}}{\sqrt{n}}>\frac{\sqrt{\varepsilon t_{n} \sqrt{n}}}{\lambda_{n}^{1 / 4} \sqrt{10 M}}\right] \leq-\lim _{n \rightarrow \infty} \frac{c_{2} k_{n}}{t_{n}^{2}}=-\infty .
\end{aligned}
$$

Also, by condition (8), since $t_{n}=\omega(1)$

$$
\lim _{n \rightarrow \infty} \frac{1}{t_{n}^{2}} \log \mathbb{P}\left[\left|W_{n}\right|>\frac{n \sqrt{\varepsilon t_{n} \sqrt{n}}}{\lambda_{n}^{1 / 4} \sqrt{10 M}}\right] \leq \lim _{n \rightarrow \infty} \frac{1}{t_{n}^{2}} \log \mathbb{P}\left[\left|W_{n}\right|>\frac{n^{5 / 4} \sqrt{t_{n}} \sqrt{\varepsilon}}{\sqrt{10 M}}\right]=-\infty .
$$

As a consequence, we have that if $t_{n}=o\left(\sqrt{k_{n}}\right)$, as $n \rightarrow \infty$, for any $\varepsilon>0$,

$$
\lim _{n \rightarrow \infty} \frac{1}{t_{n}^{2}} \log \mathbb{P}\left[\left|\frac{1}{t_{n}} \mathscr{X}_{n, p}-\mathscr{Y}_{n, p}\right|>\varepsilon\right]=-\infty .
$$

This proves that the sequences of random variables $\mathscr{X}_{n, p}$ and $\mathscr{Y}_{n, p}$ are exponentially equivalent.

To finish the proof, in view of Lemma 2.2 it is enough to argue that if $\left(n-k_{n}\right)=o\left(\frac{\sqrt{k_{n}}}{t_{n}}\right)$, then the sequences of random variables $\widetilde{\mathscr{Y}}_{n, p}$ and $\mathscr{Y}_{n, p}$ are exponentially equivalent as well. For this we recall the definition of $\zeta_{2}^{(n)}$ and write, for $\varepsilon>0$,

$$
\mathbb{P}\left[\left|\mathscr{Y}_{n, p}-\widetilde{\mathscr{Y}}_{n, p}\right|>\varepsilon\right]=\mathbb{P}\left[\left|\sum_{i=k_{n}+1}^{n}\left(g_{i}^{2}-1\right)\right|>\frac{2 \varepsilon n t_{n}}{\sqrt{k_{n}}}\right] .
$$

Denoting by $g$ a standard Gaussian random variable and applying a union bound we see that

$$
\begin{aligned}
\mathbb{P}\left[\left|\mathscr{Y}_{n, p}-\widetilde{\mathscr{Y}}_{n, p}\right|>\varepsilon\right] & \leq \mathbb{P}\left[\sum_{i=k_{n}+1}^{n} g_{i}^{2}>\frac{2 \varepsilon n t_{n}}{\sqrt{k_{n}}}-\left(n-k_{n}\right)\right] \\
& \leq\left(n-k_{n}\right) \mathbb{P}\left[g>\sqrt{\frac{2 \varepsilon n t_{n}}{\left(n-k_{n}\right) \sqrt{k_{n}}}-1}\right] .
\end{aligned}
$$

But since $\mathbb{P}[g>x] \leq(x \sqrt{2 \pi})^{-1} e^{-x^{2} / 2}$ for any $x>0$, we conclude that

$$
\lim _{n \rightarrow \infty} \frac{1}{t_{n}^{2}} \mathbb{P}\left[\left|\mathscr{Y}_{n, p}-\widetilde{\mathscr{Y}}_{n, p}\right|>\varepsilon\right] \leq-\lim _{n \rightarrow \infty} \frac{1}{t_{n}^{2}} \frac{2 \varepsilon n t_{n}}{\left(n-k_{n}\right) \sqrt{k_{n}}}=-\lim _{n \rightarrow \infty} \frac{2 \varepsilon}{n-k_{n}} \frac{\sqrt{k_{n}}}{t_{n}} \frac{n}{k_{n}} .
$$

Since $n-k_{n}=o\left(\frac{\sqrt{k_{n}}}{t_{n}}\right)$, the last expression tends to $-\infty$, as $n \rightarrow \infty$. This proves the desired exponential equivalence.

Acknowledgement. Parts of the research presented in this work have been carried out during the workshop New Perspectives and Computational Challenges in High Dimensions that took place at the Mathematical Research Institute Oberwolfach (MFO) in February 2020. All support for providing an excellent atmosphere and working conditions is gratefully acknowledged. JP is supported by the Austrian Science Fund (FWF) Project P32405 "Asymptotic Geometric Analysis and Applications".

\section{REFERENCES}

[1] D. Alonso-Gutiérrez and J. Bastero. Approaching the Kannan-Lovász-Simonovits and variance conjectures, volume 2131 of Lecture Notes in Mathematics. Springer, Cham, 2015.

[2] D. Alonso-Gutiérrez, J. Prochno, and C. Thäle. Large deviations for high-dimensional random projections of $\ell_{p}^{n}$-balls. Adv. in Appl. Math., 99:1-35, 2018.

[3] D. Alonso-Gutiérrez, J. Prochno, and C. Thäle. Gaussian fluctuations for high-dimensional random projections of $\ell_{p}^{n}$-balls. Bernoulli, 25(4A):3139-3174, 2019.

[4] I. Bárány and Z. Füredi. Computing the volume is difficult. Discrete Comput. Geom., 2(4):319-326, 1987.

[5] F. Barthe, O. Guédon, S. Mendelson, and A. Naor. A probabilistic approach to the geometry of the $\ell_{p}^{n}$-ball. Ann. Probab., 33(2):480-513, 2005. 
[6] F. Barthe and P. Wolff. Remarks on non-interacting conservative spin systems: the case of gamma distributions. Stochastic Process. Appl., 119(8):2711-2723, 2009.

[7] S. Bobkov and C. Houdré. Some connections between isoperimetric and Sobolev-type inequalities. Mem. Amer. Math. Soc., 129(616):viii+111, 1997.

[8] S. G. Bobkov. Spectral gap and concentration for some spherically symmetric probability measures. In Geometric aspects of functional analysis, volume 1807 of Lecture Notes in Math., pages 37-43. Springer, Berlin, 2003.

[9] S.G. Bobkov. On isoperimetric constants for log-concave probability distributions. In Geometric Aspects of Functional Analysis, volume 1910 of Lecture Notes in Math., pages 81-88. Springer, Berlin, 2007.

[10] H. Cramér. Sur un nouveau théorème-limite de la théorie des probabilités. Actual. sci. industr. (Confér. internat. Sci. math. Univ. Genève. Théorie des probabilités. III: Les sommes et les fonctions de variables aléatoires.), 736:5-23, 1938.

[11] A. Dembo and O. Zeitouni. Large Deviations. Techniques and Applications, volume 38 of Stochastic Modelling and Applied Probability. Springer-Verlag, Berlin, 2010. Corrected reprint of the second (1998) edition.

[12] F. den Hollander. Large Deviations, volume 14 of Fields Institute Monographs. American Mathematical Society, Providence, RI, 2000.

[13] M. Dyer, A. Frieze, and R. Kannan. A random polynomial-time algorithm for approximating the volume of convex bodies. J. ACM, 38(1):1-17, January 1991.

[14] G. Elekes. A geometric inequality and the complexity of computing volume. Discrete Comput. Geom., 1(4):289-292, 1986.

[15] N. Gantert, S.S. Kim, and K. Ramanan. Large deviations for random projections of $\ell^{p}$ balls. Ann. Probab., 45(6B):44194476, 2017.

[16] N. Gantert, K. Ramanan, and F. Rembart. Large deviations for weighted sums of stretched exponential random variables. Electron. Commun. Probab., 19:no. 41, 14, 2014.

[17] M. Gromov and V. D. Milman. Generalization of the spherical isoperimetric inequality to uniformly convex Banach spaces. Compositio Math., 62(3):263-282, 1987.

[18] J. Grote, Z. Kabluchko, and C. Thäle. Limit theorems for random simplices in high dimensions. ALEA Lat. Am. J. Probab. Math. Stat., 16(1):141-177, 2019.

[19] N. Huet. Spectral gap for some invariant log-concave probability measures. Mathematika, 57(1):51-62, 2011.

[20] S. G. G. Johnston and J. Prochno. Berry-Esseen bounds for random projections of $\ell_{p}^{n}$-balls. arXiv e-prints, page arXiv:1911.00695, Nov 2019 .

[21] Z. Kabluchko, A. E. Litvak, and D. Zaporozhets. Mean width of regular polytopes and expected maxima of correlated Gaussian variables. Zap. Nauchn. Sem. S.-Peterburg. Otdel. Mat. Inst. Steklov. (POMI), 442(Veroyatnost/ i Statistika. 23):75-96, 2015.

[22] Z. Kabluchko, J. Prochno, and C. Thäle. High-dimensional limit theorems for random vectors in $\ell_{p}^{n}$-balls. Commun. Contemp. Math., 21(1):1750092, 30, 2019.

[23] Z. Kabluchko, J. Prochno, and C. Thäle. High-dimensional limit theorems for random vectors in $\ell_{p}^{n}$-balls. II. Commun. Contemp. Math. (to appear), 2019+.

[24] Z. Kabluchko, J. Prochno, and C. Thäle. Sanov-type large deviations in Schatten classes. Ann. Inst. Henri Poincaré Probab. Stat., 56(2):928-953, 2020.

[25] O. Kallenberg. Foundations of Modern Probability. Probability and its Applications. Springer-Verlag, New York, second edition, 2002.

[26] R. Kannan, L. Lovász, and M. Simonovits. Isoperimetric problems for convex bodies and a localization lemma. Discrete Comput. Geom., 13(3-4):541-559, 1995.

[27] S.S. Kim and K. Ramanan. A conditional limit theorem for high-dimensional $\ell^{p}$-spheres. J. Appl. Probab., 55(4):1060-1077, 2018.

[28] B. Klartag. A central limit theorem for convex sets. Invent. Math., 168(1):91-131, 2007.

[29] B. Klartag. Poincaré inequalities and moment maps. Ann. Fac. Sci. Toulouse Math. (6), 22(1):1-41, 2013.

[30] R. Latał a and J. O. Wojtaszczyk. On the infimum convolution inequality. Studia Math., 189(2):147-187, 2008.

[31] M. Ledoux. A simple analytic proof of an inequality by P. Buser. Proc. Amer. Math. Soc., 121(3):951-959, 1994.

[32] Y. T. Lee and S. S. Vempala. Eldan's stochastic localization and the KLS hyperplane conjecture: an improved lower bound for expansion. In 58th Annual IEEE Symposium on Foundations of Computer Science-FOCS 2017, pages 998-1007. IEEE Computer Soc., Los Alamitos, CA, 2017.

[33] Y.-T. Liao and K. Ramanan. Geometric sharp large deviations for random projections of $\ell_{p}^{n}$ spheres. arXiv e-prints, page arXiv:2001.04053, January 2020.

[34] G. Paouris, P. Pivovarov, and J. Zinn. A central limit theorem for projections of the cube. Probab. Theory Related Fields, 159(3-4):701-719, 2014.

[35] M. Schmuckenschläger. CLT and the volume of intersections of $l_{p}^{n}$-balls. Geom. Dedicata, 85(1-3):189-195, 2001.

[36] S. Sodin. An isoperimetric inequality on the $l_{p}$ balls. Ann. Inst. Henri Poincaré Probab. Stat., 44(2):362-373, 2008.

[37] S. Soojin Kim and K. Ramanan. An asymptotic thin shell condition and large deviations for multidimensional projections. arXiv e-prints, page arXiv:1912.13447, December 2019.

[38] S. S. Vempala. Recent progress and open problems in algorithmic convex geometry. In 30th International Conference on Foundations of Software Technology and Theoretical Computer Science, volume 8 of LIPIcs. Leibniz Int. Proc. Inform., pages 42-64. Schloss Dagstuhl. Leibniz-Zent. Inform., Wadern, 2010. 
Departamento de Matemáticas, Universidad de Zaragoza, Spain

Email address: alonsod@unizar.es

Institut für Mathematik \& Wissenschaftliches Rechnen, Karl-Franzens-Universität Graz, Austria

Email address: joscha.prochno@uni-graz.at

Faculty of Mathematics, Ruhr University Bochum, Germany

Email address: christoph.thaele@rub.de 